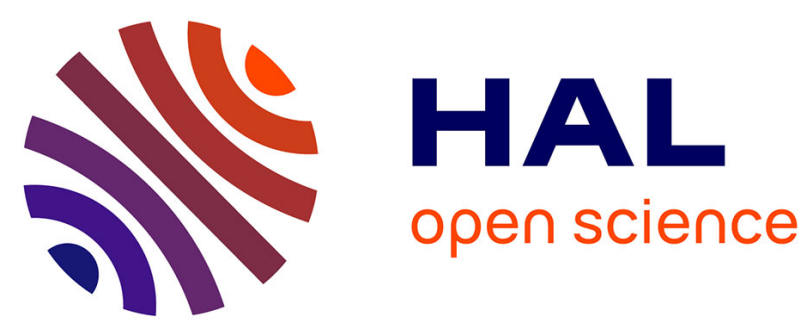

\title{
A study of the properties of a local dust storm with Mars Express OMEGA and PFS data
}

Anni Määttänen, Thierry Fouchet, O. Forni, François Forget, H. Savijärvi, Brigitte Gondet, R. Melchiorri, Yves Langevin, V. Formisano, M. Giuranna, et al.

\section{To cite this version:}

Anni Määttänen, Thierry Fouchet, O. Forni, François Forget, H. Savijärvi, et al.. A study of the properties of a local dust storm with Mars Express OMEGA and PFS data. Icarus, 2009, 201 (2), pp.504-516. 10.1016/j.icarus.2009.01.024 . hal-00533504

\section{HAL Id: hal-00533504 https://hal.science/hal-00533504}

Submitted on 7 Nov 2010

HAL is a multi-disciplinary open access archive for the deposit and dissemination of scientific research documents, whether they are published or not. The documents may come from teaching and research institutions in France or abroad, or from public or private research centers.
L'archive ouverte pluridisciplinaire HAL, est destinée au dépôt et à la diffusion de documents scientifiques de niveau recherche, publiés ou non, émanant des établissements d'enseignement et de recherche français ou étrangers, des laboratoires publics ou privés. 


\section{Accepted Manuscript}

A study of the properties of a local dust storm with Mars Express OMEGA and PFS data

A. Määttänen, T. Fouchet, O. Forni, F. Forget, H. Savijärvi,

B. Gondet, R. Melchiorri, Y. Langevin, V. Formisano, M. Giuranna,

J.-P. Bibring

PII: $\quad$ S0019-1035(09)00047-5

DOI: $\quad$ 10.1016/j.icarus.2009.01.024

Reference: $\quad$ YICAR 8907

To appear in: Icarus

Received date: 11 April 2008

Revised date: 6 November 2008

Accepted date: 21 January 2009

Please cite this article as: A. Määttänen, T. Fouchet, O. Forni, F. Forget, H. Savijärvi, B. Gondet, R. Melchiorri, Y. Langevin, V. Formisano, M. Giuranna, J.-P. Bibring, A study of the properties of a local dust storm with Mars Express OMEGA and PFS data, Icarus (2009), doi:

10.1016/j.icarus.2009.01.024

This is a PDF file of an unedited manuscript that has been accepted for publication. As a service to our customers we are providing this early version of the manuscript. The manuscript will undergo copyediting, typesetting, and review of the resulting proof before it is published in its final form. Please note that during the production process errors may be discovered which could affect the content, and all legal disclaimers that apply to the journal pertain. 


\title{
A study of the properties of a local dust storm with Mars Express OMEGA and PFS data
}

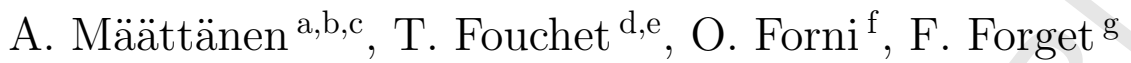 \\ H. Savijärvi ${ }^{\text {a }}$ B. Gondet ${ }^{\text {h }}$, R. Melchiorri ${ }^{\text {d,i }}$, Y. Langevin ${ }^{\text {h }}$, \\ V. Formisano ${ }^{j}$, M. Giuranna $^{\mathrm{j}}$ J.-P. Bibring $^{\mathrm{h}}$, \\ ${ }^{a}$ Department of Physics, University of Helsinki, Helsinki, Finland \\ ${ }^{\mathrm{b}}$ Space Research Unit, Finnish Meteorological Institute, Helsinki, Finland \\ ${ }^{\mathrm{c}}$ presently at: Service d'aéronomie, Verrières-le-buisson, France \\ ${ }^{\mathrm{d}}$ Observatoire de Paris, LESIA, Meudon, France \\ e Université Pierre et Marie Curie - Paris 6, UMR8109, Paris, France \\ ${ }^{\mathrm{f}}$ CESR, Toulouse, France \\ ${ }^{\mathrm{g}}$ Laboratoire de météorologie dynamique, Paris, France \\ ${ }^{\mathrm{h}}$ Institut d'astrophysique spatiale, Université Paris-sud, Orsay, France \\ ${ }^{\mathrm{i}}$ presently at: NASA/AMES, Moffett Field CA, USA

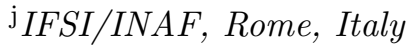

Copyright (c) 2008 A. Määttänen and authors listed above

Number of pages: 35

Number of tables: 1

Number of figures: 11

Preprint submitted to Icarus

10 February 2009 


\section{Proposed Running Head:}

Dust storm seen by MEx/OMEGA and MEx/PFS

\section{Please send Editorial Correspondence to:}

A. Määttänen

Service d'aéronomie

Réduit de Verrières - BP 3

Route des Gatines

91371 Verrières-le-buisson

France

Email: anni.maattanen@aerov.jussieu.fr

Phone: +33164474300

Fax: +33169202999 


\section{ABSTRACT}

We present observations of a local dust storm performed by the OMEGA and PFS instruments aboard Mars Express. OMEGA observations are used to retrieve the dust single-scattering albedo in the spectral range $0.4-4.0 \mu \mathrm{m}$. The single-scattering albedo shows fairly constant values between $0.6-2.6 \mu \mathrm{m}$, and a sharp decrease at wavelengths shorter than $0.6 \mu \mathrm{m}$, in agreement with previous studies. It presents a small absorption feature due to ferric oxide at $0.9 \mu \mathrm{m}$, and a strong absorption feature due to hydrated minerals between 2.7 - 3.6 $\mu \mathrm{m}$. We use a statistical method, the Independent Component Analysis, to determine that the dust spectral signature is decoupled from the surface albedo, proving that the retrieval of the single-scattering albedo is reliable, and we map the dust optical thickness with a conventional radiative transfer model. The effect of the dust storm on the atmospheric thermal structure is measured using PFS observations. We also simulate the thermal impact of the dust storm using a one-dimensional atmospheric model. A comparison of the retrieved and modeled temperature structures suggests that the dust in the storm should be confined to the 1-2 lowest scale heights of the atmosphere. However, the observed OMEGA reflectance in the $\mathrm{CO}_{2}$ absorption bands does not support this suggestion. 


\section{Introduction}

Dust is a ubiquitous component of the Martian atmosphere, and its source is the surface of the planet. Dust particles have a strong effect on circulation patterns via their impact on atmospheric heating rates (Gierasch and Goody, 1972). They also affect the global climatic cycles of volatiles $\left(\mathrm{CO}_{2}\right.$ and $\left.\mathrm{H}_{2} \mathrm{O}\right)$ via their role as cloud condensation nuclei (Michelangeli et al., 1993; Rodin et al., 1999). Dust distribution itself is affected by the atmospheric circulation, and cloud formation redistributes dust via sedimentation. These feedback mechanisms between dust distribution, radiative balance, atmospheric temperatures and circulation, and cloud formation are very nonlinear in nature. A good summary on the Martian dust cycle can be found in Kahn et al. (1992).

Mars hosts a large variety of dust phenomena, starting from the small-scale dust devils going up to planetary scale dust storm events. Surveys and classifications of storms of all scales and dust devils observed in visible spacecraft images can be found in Briggs et al. (1979), Cantor et al. (2001) and Cantor et al. (2006). In the thermal infrared, Smith (2004) have monitored the mean dust optical thickness during three consecutive Martian years, while Smith (2002) reviewed specifically the planet-encircling dust storm of 2001. However, the two visible surveys (Briggs et al., 1979; Cantor et al., 2001) present discrepancies in their spatial distributions of dust storms that are currently unexplained: the Mars Orbiter Camera survey (MOC, Cantor et al. (2001)) found fewer low-latitude dust storms than the Viking survey (Briggs et al., 1979), and MOC also found most of the regional dust storms to be located in the Northern Hemisphere in contrast with the historical record. Cantor et al. (2001) explained this difference as arising from a temporal change in dust 
sources and in atmospheric conditions favorable for dust storms.

One important factor affecting the expansion of storms is the radiative effect of dust on atmospheric circulations: suspended dust warms the atmosphere and the warming enhances the circulations. However, the warming also tends to stabilize the vertical temperature profile of the atmosphere, suppress convection, and smooth out horizontal temperature gradients inside the storm. In the end Cantor et al. (2001) pointed out that this effect can lead to a decay of the storm via a self-destructive mechanism. However, mechanical turbulence caused by wind shear can sustain dust lifting even in an isothermal situation, if the surface winds are strong. Modeling studies of the Martian dust cycle have been conducted by, e.g., Murphy et al. (1995); Newman et al. (2002a,b); Basu et al. (2004, 2006); Kahre et al. (2005, 2006), and Kahre et al. (2008).

The radiative effects of dust are dictated by the size, composition, and optical properties of the dust particles. Several authors using a multitude of observations have analyzed these properties in detail or partly, depending, for example, on the wavelength range of the instrument used, and the type of the used observations (Pollack et al., 1979; Ockert-Bell et al., 1997; Forget, 1998; Tomasko et al., 1999; Markiewicz et al., 1999; Fedorova et al., 2002; Clancy et al., 2003; Wolff and Clancy, 2003; Mateshvili et al., 2007; Vincendon et al., 2007). The frequently used single-scattering properties of dust in near-infrared are those presented by Ockert-Bell et al. (1997). They, however, did not present any values in the $2.5-2.9 \mu \mathrm{m} \mathrm{CO} 2$ band, since they were not able to retrieve the surface albedo at these wavelengths. Within this band, the analysis of Fedorova et al. (2002) revealed a strong absorption feature centered at $2.8 \mu \mathrm{m}$. Fedorova et al. (2002) attributed this absorption to the presence of phyllosilicates in the dust. Indeed, new observations from Mars Express and 
Mars Odyssey have shown the presence of phyllosilicates also on the surface of the planet (Bibring et al., 2005; Poulet et al., 2005; Chevrier et al., 2007).

In this study we present the analysis of a local dust storm observed by two Mars Express instruments. OMEGA (Observatoire pour la minéralogie, l'eau, les glaces et l'activité) was used to retrieve the dust single-scattering albedo in the range $0.4-4.0$ micron, and to determine the structure of the optical thickness within the dust storm. The Planetary Fourier Spectrometer (PFS) was used to determine the impact of the dust storm on the atmospheric temperatures. We also compared these data with 1-D atmospheric model calculations modeling the effect of dust on the atmospheric temperature field.

Section 2 introduces the instruments and the data. Then, in Section 3, we present the different methods used to identify the dust storm in the OMEGA and PFS data. Section 4 presents the method for retrieving the single-scattering albedo as well as mapping the dust optical thickness, and discusses the results. Section 5 introduces the methods of retrieval and retrieved temperatures from the PFS data, discusses the temperature structure inside the storm, and compares the results to model runs with a 1-D atmospheric column model for Mars. In Section 6, we summarize the results.

\section{Observations}

Mars Express orbiter was successfully inserted into Martian orbit on December 25th, $2003\left(\mathrm{Ls}=322^{\circ}\right)$. The satellite is located on an eccentric quasi-polar orbit (inclination of $86.35^{\circ}$ ). The periapsis altitude is approximately $250 \mathrm{~km}$, while the periapsis latitude has drifted during the mission from close to equator at 
the beginning of the mission to over the South Pole in July 2004 (Orbit \#523, Ls $=48.8^{\circ}$ ), and then northward to reach the North Pole in April 2005 (Orbit $\left.\# 1536, \mathrm{Ls}=183.4^{\circ}\right)$. The spatial resolution obtained at a given latitude hence depends on the orbit or season of the observations.

In this section, we present the two Mars Express instruments involved in this study, we briefly discuss the calibration of their data, and we describe the data selection process.

\subsection{The instruments}

\subsubsection{The OMEGA spectro-imaging instrument and calibration of data}

OMEGA is a visible and near-IR mapping spectrometer, with coaligned channels working in the $0.38-1.05 \mu \mathrm{m}$ visible and near-IR range (VNIR channel) and in the $0.93-5.1 \mu \mathrm{m}$ short wavelength IR range (SWIR channel). The data products constitute three-dimensional $(\mathrm{x}, \mathrm{y}, \lambda)$ image-cubes, with two spatial and one spectral dimensions. The spectral resolution is $7 \mathrm{~nm}$ in the VNIR channel, $14 \mathrm{~nm}$ in the $0.93-2.73 \mu \mathrm{m}$ and $28 \mathrm{~nm}$ in the $2.55-5.1 \mu \mathrm{m}$ range. An extensive description of OMEGA can be found in Bibring et al. (2004).

OMEGA's photometric calibration was carried out in a laboratory using a tunable black body with temperature stabilized up to $1200^{\circ} \mathrm{C}$ and a cold black body (down to $70 \mathrm{~K}$ ). Additional in-flight calibration were carried out by comparing previous observations and models of Phobos reflectance with OMEGA observations. The absolute photometric calibration is reliable down to the $15 \%$ level, while the relative calibration between two spectra is better than $5 \%$. The typical integration time, defined by the spacecraft ground-track 
velocity and the spatial sampling chosen, is $2.5 \mathrm{~ms}$ or $5 \mathrm{~ms}$. With such integration times, a signal-to-noise ratio of higher than 100 is obtained over the entire infrared spectral range, and about 50 in the VNIR channel.

\subsubsection{The PFS spectrometer and calibration of data}

The Planetary Fourier Spectrometer is an infrared double-pendulum interferometer working in two different channels. The Short Wavelength Channel (SWC) covers wavenumbers from 1700 to $8200 \mathrm{~cm}^{-1}(1.2-5.9 \mu \mathrm{m})$ while the Long Wavelength Channel (LWC) operates in the range $250-1700 \mathrm{~cm}^{-1}$ $(5.9-40 \mu \mathrm{m})$. In the present analysis, we have used data from LWC only. The LWC is characterized by an unapodized spectral resolution of $1.3 \mathrm{~cm}^{-1}$, a sampling step of $1.0 \mathrm{~cm}^{-1}$ and a field of view of $50 \mathrm{mrad}$ (FWHM). This corresponds to $12 \mathrm{~km}$ on Mars surface when viewed from periapsis. A more detailed description of the PFS instrument can be found in Formisano et al. (2005).

The PFS-LW channel calibration has been presented in detail by Giuranna et al. (2005). Within the wavenumber range used to retrieve the temperature profile $\left(500-800 \mathrm{~cm}^{-1}\right)$, the noise equivalent spectral radiance (NESR) of a single spectrum varies between $7 \times 10^{-8}$ and $1.5 \times 10^{-7} \mathrm{~W} \mathrm{~cm}{ }^{-2} \mathrm{sr}^{-1} / \mathrm{cm}^{-1}$, corresponding to a signal-to-noise ratio of 50 - 100 on a mid-latitude daytime spectrum. 


\subsection{Data selection}

In the beginning of the mission the Mars Express ground-tracks overlapped each other every eleven orbits, i.e. three Martian days apart (later on the resonance has been changed). This property allowed for searching rapid temporal changes in the Martian spectrum that could be attributed to dust lifting events over a limited geographical area (see Section 3 for the spectral criteria). Within a few of such events, we chose two orbits, \#1201 and \#1212 (Ls = $135^{\circ}$, MY27), which overlap south of the equator (Fig. ??). The overlap was focused on observing a surface feature in the Pollack crater and subsequently provided us with a dataset suitable for studying transient atmospheric features. Such features were found, since the latter orbit, \#1212, showed strong evidence, in both OMEGA and PFS spectra, for the presence of an optically thick, local dust storm centered at $3^{\circ} \mathrm{S}, 24.7^{\circ} \mathrm{E}$, at $\mathrm{L}_{s}=135^{\circ}$ and 13.30 local time.

[Figure 1]

\section{Identification of the local dust storm}

\subsection{Spectroscopic recognition of atmospheric dust}

Figure ??, panels a and b, compares the OMEGA spectra obtained by averaging of pixels common to both orbits \#1201 and \#1212 between $2^{\circ} \mathrm{S}$ and $4^{\circ} \mathrm{S}$. In the solar-reflected part of the spectra, the reflectance factor in the $1-2.5 \mu \mathrm{m}$ range rises from $\sim 0.3$ for the orbit \#1201 to about 0.40 for the 
orbit \#1212, while it increases by about $\sim 0.05$ in the $2.8-3.5 \mu \mathrm{m}$ range. Still in the solar-reflected range, the contrast (ratio of the band depth to the continuum level) of the strong $\mathrm{CO}_{2}$ bands at 2.7 and $2.0 \mu \mathrm{m}$ decreases from the former orbit to the latter, while the weak $\mathrm{CO}_{2}$ bands at 1.6 and $1.4 \mu \mathrm{m}$ completely disappear in the spectrum obtained in orbit \#1212. In the thermal infrared, at wavelengths longer than $4.4 \mu \mathrm{m}$, the brightness temperature decreases from $275 \mathrm{~K}$ (\#1201) down to $235 \mathrm{~K}$ (\#1212). There is also a hint of an atmospheric warming revealed by the increased brightness temperature within the $\mathrm{CO}_{2}$ band at $4.35 \mu \mathrm{m}$.

[Figure 2]

These spectral differences between OMEGA spectra recorded about three Martian days apart can only be attributed to the presence of suspended atmospheric dust at the orbit \#1212. The Martian dust is known to be bright, with large single-scattering albedo (Ockert-Bell et al., 1997). The presence of atmospheric dust above low-albedo terrains thus increases the observed reflectance. The decrease in contrast of the $\mathrm{CO}_{2}$ bands can also be attributed to the presence of dust. In fact, the reflectance factor in the core of the $\mathrm{CO}_{2}$ 2.7- $\mu \mathrm{m}$ absorption band constitutes one of the best indicators of the presence of atmospheric dust. The core of this band is saturated for surface pressures greater than $200-300 \mathrm{~Pa}$ (Titov et al., 2000), which means that all radiation is absorbed by the $\mathrm{CO}_{2}$ before reaching the surface and none escapes to space: thus the reflection equals zero in the band center if the atmosphere is clear of scatterers. Inside a saturated band, the observed reflection is nearly insensitive to surface albedo. If any reflection is seen in the band center, it must come from atmospheric aerosol particles that scatter the radiation before it is absorbed by $\mathrm{CO}_{2}$. The same effect explains the decrease in contrast of the 
$\mathrm{CO}_{2}$ absorption bands centered at 1.4, 1.6, and $2.0 \mu \mathrm{m}$, although these bands are not saturated. Finally, the decrease in the brightness temperature at wavelengths longer than $4.4 \mu \mathrm{m}$ can be explained by a combination of two effects: first, the surface temperature decreases as the atmospheric dust absorbs the solar flux, and second, the thermal emission observed during an optically thick dust storm comes from atmospheric levels located at high altitudes, where the temperatures are colder than at the surface.

In Fig. ??, we compare the PFS spectra taken during the two orbits at $24.7^{\circ}$ $\mathrm{E}$ of longitude and between $4^{\circ} \mathrm{S}$ and $2^{\circ} \mathrm{S}$. The brightness temperature of the continuum outside the $\mathrm{CO}_{2}$ band centered at $668 \mathrm{~cm}^{-1}$ decreases by about 70 K from orbit \#1201 to orbit \#1212. In contrast, the brightness temperature in the core of the $\mathrm{CO}_{2}$ band increases by about $10 \mathrm{~K}$. Again, this behavior of the thermal spectrum is typical of the occurrence of a local dust storm which cools the surface and the lower atmosphere while it heats up the midatmosphere. The Martian dust presents an absorption feature at $1100 \mathrm{~cm}^{-1}$ due to a silicate vibration mode (Smith et al., 2000). Unfortunately, this absorption feature cannot be detected on orbit \#1212 as the PFS spectrum in dusty conditions is too noisy in the range $1000-1300 \mathrm{~cm}^{-1}$. Therefore, we are not able to measure the dust optical depth in the solar reflected and thermal range simultaneously.

[Figure 3] 


\subsection{Independent Component Analysis}

As discussed in the previous section, the spectra obtained during the orbit \#1212 from both OMEGA and PFS-LW clearly show the presence of a local dust storm between $4^{\circ} \mathrm{S}$ and $2^{\circ} \mathrm{S}$. However, we will be in position to retrieve the dust parameters from the OMEGA spectrum only if the measured reflectance is independent of the surface albedo. In order to assess this possibility, we have performed an Independent Component Analysis (ICA) on the OMEGA cubes centered on the region of interest. ICA is a method that can retrieve the separate sources that, mixed together, constitute the observed signal. The method is described in detail in the Appendix A. Our goals were: i) to separate the dust source from the surface albedo source and to estimate their relative contribution to the OMEGA spectra, ii) to map the pixels were the dust source dominates the observed signal, hence to map the geographical extension of the dust storm. We applied the ICA method to the logarithm of the observed reflectance during orbit \#1212 in the spectral range 2.6 $3.0 \mu \mathrm{m}$. Our choice of the logarithm of the observed reflectance is justified by the fact that the dust reaches high altitudes, where the growth of the $\mathrm{CO}_{2}$ transmissivity is linear in the logarithmic scale. The use of the logarithm of the reflectance also enhances the noise, but since the signal in the core of $\mathrm{CO}_{2}$ band is high due to dust scattering, this does not pose problems in our case.

[Figures 4]

Figure ?? shows a map of the two major components of the ICA decomposition of orbit \#1212: i) the first component (left panel) corresponds to the dust storm in the middle of the map, ii) the second component (right panel) 
corresponds to the surface albedo. We estimated the contributions of the different components to the observed signal by using the quadratic mean of the mixing coefficients. For the ten brightest pixels in the core of the dust storm, the dust component contributes to $99 \%$ of the power. Within the whole dust storm, the dust component contributes to about $85 \%$ of the total observed signal. Hence, the ICA method proves that spectra within the core of the dust storm are independent of the second major component, i.e. the surface albedo, and thus our single-scattering analysis will be valid.

\section{Optical properties of dust}

\subsection{Radiative transfer code}

We have modeled the OMEGA spectrum of the local dust storm in the solar-

reflected range using a radiative transfer code including the gaseous absorption of $\mathrm{CO}_{2}, \mathrm{CO}$ and $\mathrm{H}_{2} \mathrm{O}$ and taking into account the multiple scattering events. Although $\mathrm{CO}$ and $\mathrm{H}_{2} \mathrm{O}$ are minor absorbers, their bands could be misinterpreted as dust mineralogical signatures if they were not included in the calculation. Multiple scattering was handled using a two-stream approximation (Meador and Weaver, 1980). The gaseous absorptions were first calculated in a line-by-line approach using the spectrometric parameters extracted from the GEISA databank (Jacquinet-Husson et al., 1999). The line-by-line opacities were then used to calculate correlated- $k$ distributions. The modeled atmosphere was divided in 45 layers equally spaced in $\log$ (pressure). For each layer we specified in entry the gaseous abundance, the dust optical thickness at $1 \mu \mathrm{m}$, and the single-scattering albedo, the asymmetry parameter and the 
extinction cross-section as a function of wavelength. The gaseous abundances were set to the standard volume mixing ratios of 0.95 and $8 \times 10^{-4}$ for $\mathrm{CO}_{2}$ and $\mathrm{CO}$, and $1.5 \times 10^{-4}$ for $\mathrm{H}_{2} \mathrm{O}$ as derived from PFS observations on orbits 1201 and 1212 by Fouchet et al. (2007).

\subsection{Retrieval methods}

\subsubsection{Single-scattering albedo}

Our analysis method for the dust optical properties stems from the practically complete decoupling of the surface and the dust reflectance in the case of a very thick dust storm. We first averaged the ten pixels with the highest reflectance in the SWC located within the dust storm (as identified by the ICA method). Then each spectel (i.e. spectral individual element) of the averaged spectrum was fitted by adjusting only the dust single-scattering albedo. Except for the dust optical thickness, all other parameters remained fixed to the following values:

- The surface albedo was retrieved from the overlapping orbit \#1201, assuming a dust-free atmosphere $(\tau=0.0)$.

- The $g$ and $Q_{\text {ext }}$ dust parameters were fixed to the Ockert-Bell et al. (1997) values interpolated to the OMEGA spectral resolution.

- The temperature profile was retrieved from PFS data orbit \#1201 (see section 5.2).

The single-scattering albedo was determined for several different dust optical thicknesses at $1 \mu \mathrm{m}$. As shown in Fig. ??, for $\tau=5$ or $\tau=20$, the retrieved $\omega_{0}$ showed an artifact, either positive or negative, of the $\mathrm{CO}_{2}$ gaseous 
band at $2.0 \mu \mathrm{m}$. This unrealistic behavior for dust spectral properties allowed us to determine the dust optical thickness for the averaged spectrum. A single-scattering albedo devoid of $\mathrm{CO}_{2}$ band residuals was obtained for $\tau=10$ (Fig. ??), which corresponds to the highest dust optical thickness in the core of the dust storm.

[Figure 5]

We have estimated the sensitivity of our retrieved $\omega_{0}$ to several parameters:

- The surface albedo was determined assuming a dust-free atmosphere $(\tau=$ 0.0). This assumption is supported by Spiga et al. (2007), who analyzed the 2.0- $\mu \mathrm{m} \mathrm{CO}_{2}$ band to determine a low dust content of $\tau=0.1-0.2$. However, the Mars Exploration Rover (MER) observations yielded a dust content of $\tau=0.6$ at the time of the orbit 1201 of Mars Express (Wolff et al., 2006).

We calculated that the higher dust content would lead to a $5 \%$ change in the retrieved surface albedo compared to the dust-free case, leading to a change of less than $0.1 \%$ in the determination of $\omega_{0}$. This analysis provides quantitative evidence for the decoupling of the reflectance from the surface albedo whenever the dust optical thickness is large, hence validating our method.

- We assumed an asymmetry parameter of $g=0.63$, as suggested by most studies (Ockert-Bell et al., 1997; Clancy et al., 2003). This corresponds to particles of about $1 \mu \mathrm{m}$ in radius. It may vary within a few percent, but as shown by the right panel of Fig. ?? this would affect our retrieved singlescattering albedo less than $0.5 \%$.

- For the analysis we used a temperature profile determined from the dustfree orbit \#1201. This may not correspond to the atmospheric conditions 
prevailing in the core of the dust storm. However, using warmer profiles, we found that our retrieved single-scattering albedo is insensitive within $0.1 \%$ to the assumed temperature profile.

- Our method to take into account the multiple scattering events, the twostream method, is relatively simple and may not be the most accurate. We have compared the reflectance calculated with our method with the simulations made for a standard Mars atmosphere by (Tran and Rannou, 2004, http://tttrung.free.fr/) using 3D spherical Monte-Carlo algorithm, which were intended to produce as realistic results as possible. We found our calculations to lie within $5 \%$ of the true reflectance, which leads to an error of ${ }_{-0.002}^{+0.004}$ on $\omega_{0}$. Moreover, Vincendon et al. (2007) also determined the dust single-scattering albedo from the same OMEGA data, but using the 'infinite' dust optical depth approximation with a Monte-Carlo algorithm. Their $\omega_{0}$ values differ from ours by less than a percent.

- The signal-to-noise ratio for the high reflectance spectra used in this study is larger that 200. Hence, the largest error source comes from the uncertainty on the OMEGA absolute calibration, estimated to lie within $\pm 15 \%$. As shown on the right panel of Fig. ??, this corresponds to an uncertainty on the determination of the dust single-scattering albedo of ${ }_{-0.03}^{+0.02}$ in the center of $2.9 \mu \mathrm{m}$ band and ${ }_{-0.006}^{+0.012}$ in the rest of the spectral interval.

Finally, adding quadratically all the errors, our uncertainty on $\omega_{0}$ amounts to ${ }_{-0.031}^{+0.021}$ in the center of $2.9 \mu \mathrm{m}$ band and to ${ }_{-0.008}^{+0.014}$ in the rest of the spectral interval. 


\subsubsection{Dust optical thickness}

Outside the core of the dust storm, the reflectance varies with the pixels. This variation cannot be attributed to a change in the dust properties, but rather to a change in the dust optical thickness: surface and dust contributions to the reflectance can no longer be considered decoupled. Using the surface albedo retrieved from orbit 1201, this situation allows us to map the optical thickness outside the core of the dust storm. We proceeded as follows:

- We used the dust single-scattering albedo retrieved previously.

- The surface pressure was calculated for each pixel using the European Mars Climate Database, EMCD (http://www-mars.lmd.jussieu.fr/, Lewis et al. (1999)). Since the spatial grid of the EMCD is much coarser than the resolution of the OMEGA images, the pressure was hydrostatically extrapolated to each pixel using the MOLA topography.

- The surface albedo was retrieved from observations obtained during the orbit 1201. The observed area was divided in three albedo regions: low, intermediate (with a strong gradient), and high. The region-averaged values at $1 \mu \mathrm{m}$ were 0.16 (low), 0.35 (intermediate), and 0.37 (high). The areas were limited by latitudes $4.7^{\circ} \mathrm{S}$ and $0.4^{\circ} \mathrm{S}$.

- Then, finally the optical depth was adjusted to fit the observed reflectance for every wavelength of each pixel.

The errors in the determination of the dust optical thickness can be due to small-scale variations of the surface albedo and to the propagation of uncertainties in the dust single-scattering albedo $\omega_{0}$. We have estimated the smallscale variations of the surface albedo by calculating the standard deviation of the observed reflectance in the three albedo regions. The resulting uncertain- 
ties depends on the dust optical thickness. At the low-end limit, dust opacities lower than $\tau=1.0$ for the low albedo region, $\tau=2.3$ for the intermediate, and $\tau=3.0$ for the high albedo region cannot be distinguished from small-scale surface albedo variations. They hence constitute our lower limits of sensitivity. For larger opacities, the errors caused by the surface albedo decrease rapidly with $\tau: \pm 0.2$ for $\tau=4, \pm 0.1$ for $\tau=6$, and are negligible for $\tau>7$.

The uncertainties on the OMEGA absolute calibration, and the accuracy of the two-stream method propagate into uncertainties in the retrieved singlescattering albedo $\omega_{0}$. This also causes an uncertainty in the dust optical thickness, which increases with decreasing $\tau$. We have calculated this error to be ${ }_{-0.7}^{+0.5}$ at $\tau=8$, quite constant, ${ }_{-0.9}^{+0.5}$, for smaller values, and decreasing towards $\tau=10$.

Summing quadratically the errors give error bars of ${ }_{-0.9}^{+0.5}$ for $\tau$, translating to $+13.5 /-23 \%$ for $\tau=4,+8.5 /-15.2 \%$ for $\tau=6$, and $+6.75 /-11.5 \%$ for $\tau=8$. For a summary of the errors in the $\tau$ retrieval, see Table 1.

The analysis proved to be unreliable in the longer wavelength channel for two reasons. Due to the presence of thermal emission, it is difficult to retrieve the surface albedo from the orbit 1201 at wavelengths longer than $3.5 \mu \mathrm{m}$. In addition, in orbit 1212, thermal emission will also contribute significantly to the spectrum as the optical depth decreases. Around $3 \mu \mathrm{m}$, the atmospheric dust and the surface reflectivity are very similar, preventing us from separating surface and atmospheric dust contributions. Thus we are unable to distinguish variation of the surface albedo from variation of the dust optical depth at longer wavelengths, and our dust optical thickness retrieval is not reliable above $3 \mu \mathrm{m}$. 


\subsection{Results}

\subsubsection{Single-scattering albedo}

[Figure 6]

The retrieved single-scattering albedo is shown in left panel of Fig. ??. In the $0.9-2.5 \mu \mathrm{m}$ range, it agrees within $0.5 \%$ to the single-scattering albedo retrieved using the same OMEGA data by Vincendon et al. (2007). The small difference between the two studies can be accounted for by our choice of a different radiative transfer scheme than that used Vincendon et al. (2007) (two-stream against Monte-Carlo). However, our study expands the analyzed spectral range to the entire OMEGA spectrum dominated by solar reflection $(0.4-4 \mu \mathrm{m})$. In this range our retrieved values are consistent in absolute value and in spectral shape with the single-scattering albedo derived by OckertBell et al. (1997). However, our values are larger than the dust parameters retrieved from Pathfinder visible data (Tomasko et al., 1999; Markiewicz et al., 1999; Johnson et al., 2003). Particularly around $1 \mu \mathrm{m}$, this difference cannot be attributed to the 15\%-uncertainty of the OMEGA absolute calibration as shown in Fig. ??. We do not have any explanation for this disagreement. We

just note that the Pathfinder and the OMEGA dataset are not sensitive to the same parameters. The Pathfinder data are mostly sensitive to the particle mean size, while the spectral properties inferred by Tomasko et al. (1999) depend on the assumed size distribution. In contrast, the OMEGA experiment is mostly sensitive to the spectral properties and insensitive to the particle radius.

Centered at $2.9 \mu \mathrm{m}$, our retrieved single-scattering albedo shows a strong spec- 
tral feature, attributed to hydrated minerals. This is not a surprise, since this hydration band is also ubiquitous in the spectrum of Martian soils. However, the shape of our band differs from that retrieved from ISO data by Fedorova et al. (2002). Their analysis revealed a narrow absorption feature centered at $2.8 \mu \mathrm{m}$, characteristic of phyllosilicates. Phyllosilicates are also observed in OMEGA spectra (Bibring et al., 2005; Poulet et al., 2005), but on specific geographical units covering altogether a small fraction of the planet's surface. Retrieving the Martian dust single-scattering albedo in the $2.6-2.9 \mu \mathrm{m}$ strong $\mathrm{CO}_{2}$ band is difficult, since the dust scale height affects the analysis. We consider our determination of the single-scattering albedo in this spectral region more reliable than that of Fedorova et al. (2002) for the following reasons: i) ISO observations were averaged over the disk of the planet, hence encompassing regions of different dust opacities, ii) ISO observations were carried out in a period of low dust opacity when the dust should have been confined close to the surface, while our observations pertain to a local dust storm efficiently mixing dust in the first atmospheric scale heights. Indeed Fedorova et al. (2002) needed a higher single-scattering albedo at the wavelength of the strongest $\mathrm{CO}_{2}$ absorption, which points to the dust being confined close to the surface during their observations.

Besides the signature of hydrated minerals at $2.9 \mu \mathrm{m}$, the Martian dust is devoid of any mineralogical signature. There is a hint of ferric oxide absorption at $0.9 \mu \mathrm{m}$, but at the limit of the OMEGA relative calibration. The rest of the spectrum shows no spectral absorption at $0.5 \%$-level in the $1.0-2.5 \mu \mathrm{m}$ range, and at the $1 \%$-level in the $2.7-4.0 \mu \mathrm{m}$ range.

An interesting result is that altogether the Martian dust single-scattering albedo spectrum looks very similar to that of Saharan desert dust with par- 
ticle size of approximately $1 \mu \mathrm{m}$ (Tegen and Lacis, 1996). This particle size is close to the average effective radius of Martian dust, and the asymmetry parameter $g=0.63$ we have chosen for the study corresponds to particles of about this size.

\subsubsection{Dust optical depth}

The analyzed $\tau$ in the continuum at the wavelength $1 \mu \mathrm{m}$ is shown in Fig. ??. The values of the retrieved $\tau$ fall in the full range of $0.0-9.9$ in this image that is zoomed to the storm area. However, it should be kept in mind that in this albedo region our method is reliable only for $\tau>2.3$ (see Section 4.2.2). The storm shows a lot of structure in the range of $\tau$ analysed. Several optically thick, roundish dust plumes can be seen, and their diameters, when defined with the boundary $\tau=5$ (color change from blue to green in the image), vary from some kilometers to about $10 \mathrm{~km}$, although the estimates are limited by the width of the OMEGA session. The latitudinal extent of the storm area imaged in Fig. ?? is 4.24 degrees, which corresponds to about $250 \mathrm{~km}$. The width of the OMEGA image is about 0.36 degrees, corresponding to approximately $20 \mathrm{~km}$, but the storm seems to be extending outside the image, and thus we can not evaluate its exact extent in the longitudinal direction.

[Figure 7]

We have estimated a range for the lifting rate of the dust in the storm assuming that 1) the storm has started right after the previous observation at orbit \#1201, i.e. 3 sols earlier (minimum lifting rate), or that the storm started 2 hours before the observation (maximum lifting rate), and 2) that the lifted dust has not been advected away from the source region, i.e., the source region 
is the area where the optical thickness for the lifting rate estimate is calculated. For the lifting rate estimation we used the region of the storm seen by the PFS FOV for the temperature retrieval (the oval in Fig. ??) for purposes of later temperature profile comparison. The mass of the lifted dust can be estimated from the optical thickness using the relation $M=\frac{4}{3} \frac{\rho r \tau}{Q_{\text {ext }}}$, where $M$ is the dust mass in the unit column of air, $\rho$ the dust density, $r$ the particle radius, $\tau$ the dust optical thickness and $Q_{\text {ext }}$ the extinction coefficient. The values of $\tau$ and $Q_{\text {ext }}$ should be defined on the same wavelength, here at $1 \mu \mathrm{m}$, the $\rho$ was taken as $2500 \mathrm{~kg} \mathrm{~m}^{-3}$ and $r=1.5 \mu \mathrm{m}$. The average optical thickness at $1 \mu \mathrm{m}$ in the area of the PFS FOV turned out to be $\tau_{\text {avg }}=4.23$. Using the $Q_{\text {ext }}$ at $1 \mu \mathrm{m}$ from Ockert-Bell et al. (1997) the above formula gives $(0.25-9.1) \times 10^{-7} \mathrm{~kg}$ $\mathrm{m}^{-2} \mathrm{~s}^{-1}$ for the lifting rate range within the time frame of 2 hours -3 sols. For comparison, some examples of averaged lifting rates calculated from modelling are of the order of $10^{-9} \mathrm{~kg} \mathrm{~m}^{-2} \mathrm{~s}^{-1}$, (globally averaged, Basu et al., 2004), $10^{-7}-10^{-3} \mathrm{~kg} \mathrm{~m}^{-2} \mathrm{~s}^{-1}$ (zonally averaged, Kahre et al., 2008).

The storm is located on a topographical slope (highland in the south, lowland in the north) at the edge of an area of an abrupt albedo change. OMEGA data was used in retrieval of surface pressure (Forget et al., 2007; Spiga et al., 2007), and in their analysis Spiga et al. (2007) noted some occurrences of atmospheric waves via their signature in the surface pressure field. One occurrence of these waves was observed in orbit 1201, only some degrees away from the location of the dust storm observed 3 sols later. The waves occurred at a large crater centered at $8{ }^{\circ} \mathrm{S}$, whereas the storm is centered at $3{ }^{\circ} \mathrm{S}$.

Even though the dust plumes appear similar in shape as convective clouds, we can not compare the results with convective cell sizes from, for example, LES simulations (Michaels and Rafkin, 2004; Tyler et al., 2008): convection 
in such simulations has been traditionally modeled in low-dust situations, and even though such simulations do produce vortices that could lift dust (dust devils), they do not initiate dust storms. Comparison with global modelling (Murphy et al., 1995; Newman et al., 2002a,b; Basu et al., 2004, 2006; Kahre et al., 2005, 2006, 2008) is difficult because such studies focus on initiation mechanisms, details of the dust lifting parameterizations, and modeled lifting rates, but do not study the smaller scale structure of the storms in more detail, naturally also in lack of resolution. Toigo et al. (2002) modeled observed dust lifting events in the southern polar areas, but focused on the aforementioned features as well.

Thus we conclude that to study further the structure of the storm, and unveil the mechanisms responsible for it, a high-resolution mesoscale model study is required, and even though it is out of the scope of this paper, our analysis gives very good constraints for such a study.

\section{The effect of dust on the atmosphere}

\subsection{The temperature structure analysis from PFS observations}

The temperature structure retrieval is based on the fitting of the $\nu_{2}$ band of $\mathrm{CO}_{2}$ centered at $667 \mathrm{~cm}^{-1}$ as described in detail in Fouchet et al. (2007). The forward radiative transfer modeling is carried out using a line-by-line model including the gaseous absorption by $\mathrm{CO}_{2}$, but without taking dust into account since we cannot determine the dust opacity from the $9 \mu \mathrm{m}$ band. In large optical depth conditions, this will tend to underestimate the temperature in the lower atmosphere. The inversion of the temperature profile is 
carried out using the regularization and stabilization methods described by Fouchet et al. (2007). Using this technique, vertical temperature profiles can be retrieved from the surface up to a pressure level of $10 \mathrm{~Pa}(\sim 35 \mathrm{~km})$ with a vertical resolution of about one scale height. The uncertainties on the retrieved temperature for a single PFS spectrum typically amount to $\sim 2 \mathrm{~K}$ from 10 to $35 \mathrm{~km}$, but increase in the first scale height above the surface to reach about $\sim 5 \mathrm{~K}$ at the deepest pressure level.

\subsection{Retrieved PFS temperature profiles}

Figure ?? presents the latitude-pressure cross-sections of the temperature structures inverted for orbit \#1201 and \#1212. Except in the local dust storm area, the two cross-sections are quite consistent with each other at pressures larger than $70 \mathrm{~Pa}$. They are also consistent with the predictions of the LMD General Circulation Model (Forget et al., 1999) retrieved from the European Mars Climate Database, EMCD (http://www-mars.lmd.jussieu.fr/, Lewis et al. (1999)) with atmospheric temperatures of $230 \mathrm{~K}$ at tropical latitudes in the lower scale height, and of $180 \mathrm{~K}$ at the $70-\mathrm{Pa}$ pressure level. At pressures lower than $70 \mathrm{~Pa}$, the two temperature structures differ in the Southern Hemisphere. At $30^{\circ} \mathrm{S}$, the temperature retrieved was $\sim 170 \mathrm{~K}$ at $30 \mathrm{~Pa}$ during the orbit\#1201 while it was over $180 \mathrm{~K}$ during the orbit \#1212. In fact, the temperature structure retrieved during orbit \#1212 was closer to the predictions of the EMCD, with the warming at high altitudes in the Southern Hemisphere corresponding to the return branch of the Hadley circulation. Possibly the difference between the two orbits indicates a temporal variability of the Hadley circulation cell. 
[Figures 8]

The thermal fingerprint of the local dust storm is evident at $3^{\circ} \mathrm{S}$ in the temperature structure of orbit \#1212, with a net cooling close to the surface and net heating in the upper atmosphere, as expected from theoretical considerations (Gierasch and Goody, 1972). In the core of the dust storm, the temperatures near the surface and at $30 \mathrm{~Pa}$, were $\sim 10 \mathrm{~K}$ colder and $\sim 5 \mathrm{~K}$ warmer, respectively, than at the surrounding unperturbed latitudes (Fig. ??). Several measurements of temperature profiles have been performed in the millimetric range or in the thermal infrared at the occasion of regional or global dust storms. Moreno et al. (2006) and Clancy et al. (2006) observed the Southern Hemisphere in its late summer season in 2003 (MY26) and 2005 (MY27) using the CO rotational lines. The temperature profiles they derived in 2003 and 2005 differ with each other, the profile obtained in 2005 showing a weaker vertical temperature gradient than in 2003. This corresponded to the breakout of a regional dust storm at the end of MY27. In more drastic conditions, Hanel et al. (1972) and Smith et al. (2002) using spacecraft monitoring of the $\mathrm{CO}_{2}$-band, and Moreno et al. (2006) in the millimetric range, found a vertical temperature profile close to isothermal during a global dust storm.

Therefore, atmospheric dust tends to homogenize the vertical temperature profile. We observe the same trend in our temperature profiles (Fig. ??), but with a weaker amplitude than for the regional or global dust storms described above.

[Figure 9] 


\subsection{Comparison with 1-D atmospheric models}

The 1-D version of the general circulation model of the Laboratoire de Météorologie Dynamique (hereinafter LMD 1-D model, Forget et al., 1999) was used to study the effect of dust on the atmospheric temperatures: the 1-D model version includes all the physical process descriptions as the global model, and an improved, more accurate radiative transfer scheme to compute the absorption and scattering of solar radiation by airborne dust, based on the Toon et al. (1989) scheme. The dust optical properties given as input for the model were those used and retrieved in this work. The model was initialized with an isothermal temperature profile with $T=200 \mathrm{~K}$ at all model layers and the results were taken from the sol 10 at 13.30 local time (the time of the observation), if not stated otherwise. The model-produced profiles were convolved with PFS weighting functions to optimize the comparison with the PFS temperature profile retrieved in the storm area (PFS FOV of the temperature retrieval is shown in Fig. ??).

We conducted a model run for the location and season of the dust storm with the average dust optical thickness of $\tau=4.23$, calculated for the area of the PFS FOV, and a well-mixed dust vertical profile with top of the dust at $55 \mathrm{~km}$. The model results at $13.30 \mathrm{LT}$ exhibit a very warm, nearly isothermal middle atmosphere (the short-dashed line in Fig. ??). There is a clear difference between the retrieved PFS temperature profile (the solid line in Fig. ??) and the profile predicted by the model (short-dashed line in Fig. ??). The warming of middle atmosphere in the PFS profile seems to be only $5 \mathrm{~K}$ and the cooling of the surface about $10 \mathrm{~K}$, whereas the model profile is significantly warmer in the middle atmosphere $(60 \mathrm{~K}$ at $1 \mathrm{~Pa})$, and remains isothermal between $1 \mathrm{~Pa}$ 
and the surface.

The radiative response time of the Martian atmosphere is quite short (shorter than the three sols between the two orbits studied here). Hence, it is unlikely that the difference between the PFS-retrieved and the modeled temperature vertical profile can be attributed to the fact the atmospheric column did not reach thermal equilibrium. This was tested by looking at the results of the first sol of the aforementioned run with dust top at $55 \mathrm{~km}$ (see Fig. ??): already the 01 LT profile of the first sol displays the warm, isothermal middle atmosphere that was seen at sol 10 in Fig. ??. So the dust heats the atmosphere very fast and it seems very unlikely that the storm would have started so recently before the observation that the atmosphere would not yet have responded to it.

[Figure 10]

[Figure 11]

The difference between the observed and modelled temperatures could rather indicate that dust vertical distribution in the dust storm observed by OMEGA is not uniform throughout the atmosphere, but that the dust is confined in the lowest levels. A new set of model runs was made with a modified dust distribution of a well-mixed $\tau=0.2$, on top of which a $\tau=4.23$ profile limited in vertical extent was embedded. The altitude of the top of the dust storm was varied and the produced profiles were compared with the retrieved PFS profile. Examples of the model profiles are shown in Fig. ??.

The dust top altitude has a notable effect on the shape of the temperature profile (see Fig. ??). All the LMD 1-D profiles with vertically heterogeneous 
dust exhibit an inversion of differing strengths near the surface, and the shape of the profile is dictated by strong warming in the dust layer. The general shape of the profiles is, however, less steep than the observed profile, and changing the dust top altitude only moves this shape with altitude. So we can get the overall values of the modeled temperature profile to agree with that observed, in particular in the middle atmosphere, where also the PFS resolution is adequate, but the slope of the profile is not fully satisfactory. The best overall agreement seems to be found at dust top altitude around $10-20 \mathrm{~km}$.

However, it should be noted that the PFS can not resolve the details of the temperature profile near the surface, so the fact that we do not see the inversion in the PFS profile does not mean it would not exist. The retrieval procedure of the PFS temperature profile suffers from the thick dust, which degrades the reliability of the retrieval, and thus the temperature vertical profile retrieved from PFS data in the core of the dust storm must be considered with caution. As mentioned before, since we cannot retrieve the dust opacity in the thermal infrared from the silicate band at $9 \mu \mathrm{m}$ due to a too low signalto-noise ratio, we inverted the temperature profile from the $\mathrm{CO}_{2}$ band for a clear atmosphere. This tends to underestimate the temperature in the lower layers. In addition, the surface temperature and the atmospheric temperature in the first half scale height are highly correlated, which hampers an accurate inversion close to the surface.

The confinement of the dust in the first scale height would be difficult to reconcile with OMEGA data. In the well-mixed infinite dust model, an optical thickness of 10 is required to fit the $2.0-\mu \mathrm{m} \mathrm{CO}_{2}$ band in the dusty spectrum of orbit 1212. If the dust is confined to the first $10 \mathrm{~km}$, an optical thickness of 
20 is required, which seems an extremely high value.

Faced with these contradictions and uncertainties, we are not in position to firmly conclude, as suggested by the 1-D atmospheric model, that the dust is confined close to the surface. Other OMEGA and PFS orbits will be analyzed to investigate this issue in a future work.

\section{Summary and conclusions}

We have used the observations of two Mars Express instruments, OMEGA and PFS, to distinguish and analyze a local dust storm near the equator of Mars in late northern summer. The same geographical unit was observed on two orbits only 3 sols apart, and the later orbit revealed a local dust storm, whereas in the first orbit the atmosphere was very clear. These two orbits were exploited to study the optical properties of the dust and the morphology of the dust storm.

Since the dust storm is optically very thick, dust reflection is decoupled from the surface albedo and all scattering comes from the dust contribution. In this case we were not limited to the $\mathrm{CO}_{2}$ absorption bands in the retrieval, but were able to derive the dust single-scattering albedo reliably throughout the wavelength range examined. The single-scattering albedo is a basic parameter required for radiative transfer codes that are used in many applications, for example in atmospheric models and data inversion. This study has provided a rare dataset of the single-scattering albedo in the visible and near-infrared with good spectral resolution. Comparison revealed that our results agree quite well with previous studies. The results show that the single-scattering albedo of this 
particular Martian dust is constant and high, 0.97, in the shorter wavelengths $(1.0-2.6 \mu \mathrm{m})$ and that there is significant absorption by hydrated minerals between 2.7 and $3.6 \mu \mathrm{m}$. However, our results do not show the hydration band of phyllosilicates that was seen by Fedorova et al. (2002). The discrepancy between the data of Fedorova et al. (2002) and this study may be, at least partly, explained by the ISO experiment mapping the full disk of Mars and the season being characterized by fairly low dust optical thickness.

We also retrieved temperature profiles from simultaneous observations of the PFS instrument and compared them with profiles acquired with 1-D atmospheric modeling with a dusty atmosphere. The model profiles and the retrieved dust storm profile from PFS reach an acceptable degree of agreement only if the dust storm is confined in the lowest 1-2 scale heights of the atmosphere. The short duration of the storm (less than 3 sols) can support this: the dust has not had the time to mix vertically higher. However, the OMEGA data do not support this hypothesis. In addition, the details of the temperature profiles can not be studied because of limited vertical reliability of the retrieved PFS profile in this situation of very high optical thickness.

We also present a novel approach for dust storm discovery, the Independent Component Analysis. In this paper we have shown that this method can be successfully used to distinguish suspended dust from the surface features, if the dust optical thickness is high enough. 


\section{A The Independent Component Analysis method}

The Independent Component Analysis is a technique that comes from developments in the Blind Source Separation (BSS) research. The goal of ICA is to estimate $h$ source signals, assumed to be stationary and independent, using $n$ observed signals $(n \geq h)$ that are independent unknown mixing of the source signals (Comon, 1990, 1992, 1994). The most common example of this problem is the "Cocktail Party" problem. In spectroscopy, it is considered that the observed spectra are linear combinations of pure sources or poles that we want to estimate. For a complex ensemble of data in which more signals contribute to the observed data, the gaussian hypothesis of the Principal Component Analysis (PCA) will not be valid in all cases. Indeed, the goal of the PCA is to maximize the dispersion, and consequently, to decorrelate the signals assuming that all the sources follow a gaussian probability function. The extraction of pure and statistically independent spectra of a melange cannot be reliably obtained with the PCA. However, ICA like PCA, tries to transform the original representative space by searching for directions in a new space, so that the resulting vectors are independent, and not only uncorrelated. The ICA is then a method of linear transformation in which the representation is the one that minimizes the statistical dependence of the components. This is achieved, using a criterion, related to the information entropy theory, that gives back the statistical independence assuming that the data follow a non-Gaussian distribution.

The use of ICA for multispectral data analysis in planetology has been firstly performed by Forni et al. (2005) on OMEGA data proving its efficiency. It has also been applied on the same data set in combination with the Bayesian 
Positive Source Separation (Schmidt, 2007). Gomez et al. (2007) have applied ICA on hyperspectral terrestrial data with the fully constrained least squares spectral mixture analysis (FLCS) to estimate the end member abundances.

\section{A.1 Hypotheses and modeling}

In a first approximation, it is assumed that the mixing is linearly instantaneous, that the noise can be neglected and that $n=h$. Be $s_{t}$ and $x_{t}$, the source and observation vectors, there is a relation such that

$$
x_{t}=A s_{t}
$$

whre $A$ is the so called "mixing" matrix, assumed to be constant and invertible. The goal is to estimate $G$, the separation or de-mixing matrix, from the only observation matrix $x_{t}$, such that $G A$ is a diagonal matrix up to a permutation. The starting hypothesis to solve this kind of problem is the independence of the sources.

In the frame of the linear model $X=A S$ there exists a very significative link between dependence, correlation and non-gaussianity. Two random variables are said independent when the knowledge of the value of one of the variables does not bring any information about the value of the other. Independence is characterised by the fact that the joint probability distribution is equal to the product of the probability distributions. Hence, independence is a stronger hypothesis than uncorrelation. In other words, two variables that are independent are uncorrelated but the opposite is not necessarily true. This means that in the search of independent component, the decorrelation is half of the job and one may seek the basis in which the data is represented by maximally 
independent processes among those bases in which the data is decorrelated. This is done by maximizing a non-Gaussianity criterion based on higher order statitistics.

A so-called "whitening" of the data is first applied so that the mixing matrix is orthogonal and hence the de-mixing matrix as well. In fact, by multiplication with the inverse of a square root of the covariance matrix of the data one obtains:

$$
\tilde{x}_{t}=\tilde{A} s_{t}
$$

with $\tilde{A}=P D^{1 / 2} P^{T} A$ where $D$ is a diagonal matrix containing the eigenvalues of the covariance matrix of $x_{t}$ and $P$ is the orthogonal matrix of the associated eigenvectors. This insures that $E\left[\tilde{x}_{t} \tilde{x}_{t}^{T}\right]=I$ is orthogonal, being $I$ the identity matrix and $E[X]$, the expectation value of the random variable $X$.

The different components of $\tilde{x}_{t}$ are decorrelated and $\tilde{x}_{t}$ is "white" Assuming that the sources $s_{t}$ have zero mean and unit variance, we obtain:

$$
E\left[\tilde{x}_{t} \tilde{x}_{t}^{T}\right]=\tilde{A} E\left[s_{t} s_{t}^{T}\right] \tilde{A}^{T}=\tilde{A} \tilde{A}^{T}=I
$$

The mixing matrix $\tilde{A}$ is orthogonal and since $\tilde{G} \tilde{A}=I, \tilde{G}$ is also orthogonal. This leads to so-called orthogonal algorithms to retrieve the de-mixing matrix $\tilde{G}$.

After whithening of the data, one is then seeking a rotation $R$ (which leaves things white) so that $\hat{s_{t}}$ defined by

$$
\hat{s_{t}}=\tilde{A}^{-1} R \hat{x_{t}}
$$


and $\hat{G}=\widehat{A^{-1}}=\tilde{A}^{-1} R$ are estimations of the sources and of the inverse of the mixing matrix.

\section{A.2 JADE}

As in Forni et al. (2005) and Erard et al. (2008), we have used the Joint Approximate Diagonalization of Eigenmatrices method (JADE) algorithm, which is an orthogonal ICA method and, like most mainstream ICA techniques, it exploits higher order statistics related to the fourth order cumulant

$$
k(X)=E\left[X^{4}\right]-3 E\left[X^{2}\right]^{2} .
$$

This estimator is relatively sensitive to the noise and the number of samples should be high enough to minimize its influence. From the matrix of sources $S$, it is possible like in the PCA, to compute $C$ the factorial coordinates or "scores" from the following equation:

$$
C=X S^{T}\left(S \cdot S^{T}\right)^{-1}
$$

Another way to do this, is to compute the correlation coefficient of the sources $S$ with the signal $X$. The advantage is that, since the sources are independent, the sum of the correlation coefficient squared is 1 . This property allows to have an estimate of the contribution of each independent component to the signal.

More details on JADE can be found in Cardoso (1998, 1999); Hyvärinen et al. (2001). 


\section{Acknowledgements}

We thank the reviewers for comments that significantly improved the paper. We thank Dr. A. Fedorova for providing the data for comparison and A. Spiga for discussions about the surface pressure values and providing the program for plotting nice maps of OMEGA images. AM wishes to thank Association Franco-Finlandaise pour Recherche Scientifique et Technique, the Wihuri foundation, the Enhrnrooth foundation and the Väisälä foundation for their financial support that made possible the visits to Paris (approximately 5 months in total) to do this work during the period Nov $2005-$ Dec 2007. Funding from the Kordelin foundation, the Graduate school in astronomy and space physics and the Centre National d'Etudes Spatiales are gratefully acknowledged.

\section{References}

Basu, S., Richardson, M. I., Wilson, R. J., 2004. Simulation of the Martian dust cycle with the GFDL Mars GCM. J. Geophys. Res. 109, E11006, doi:10.1029/2004JE002243.

Basu, S., Wilson, J., Richardson, M., Ingersoll, A., 2006. Simulation of spontaneous and variable global dust storms with the GFDL Mars GCM. Journal of Geophysical Research 111, E09004, doi:10.1029/2005JE002660.

Bibring, J.-P., Langevin, Y., Gendrin, A., Gondet, B., Poulet, F., Berth, M., Soufflot, A., Arvidson, R., Mangold, N., Mustard, J., Drossart, P., the OMEGA team, 2005. Mars surface diversity as revealed by the OMEGA/Mars Express observations. Science 307 (5715), 1576-1581, doi:10.1126/science.1108806. 
Bibring, J.-P., Soufflot, A., Berthe, M., Langevin, Y., Gondet, B., Drossart, P., Bouye, M., Combes, M., Puget, P., Semery, A., Bellucci, G., Formisano, V., Moroz, V., Kottsov, V., the OMEGA Co-I team, Bonello, G., Erard, S., Forni, O., Gendrin, A., Manaud, N., Poulet, F., Poulleau, G., Encrenaz, T., Fouchet, T., Melchiorri, R., Altieri, F., Ignatiev, N., Titov, D., Zasova, L., Coradini, A., Capacionni, F., Cerroni, P., Fonti, S., Mangold, N., Pinet, P., Schmitt, B., Sotin, C., Hauber, E., Hoffmann, H., Jaumann, R., Keller, U., Arvidson, R., Mustard, J., Forget, F., 2004. Omega: Observatoire pour la minéralogie, l'eau, les glaces et l'activité. ESA SP-1240: Mars Express: the scientific payload, 37-49.

Briggs, G. A., Baum, W. A., Barnes, J., Jun. 1979. Viking Orbiter imaging observations of dust in the Martian atmosphere. J. Geophys. Res. 84, 27952820.

Cantor, B. A., James, P. B., Caplinger, M., Wolff, M. J., 2001. Martian dust storms: 1999 Mars Orbiter Camera observations. J. Geophys. Res. 106 (E10), 23653-23687.

Cantor, B. A., Kanak, K. M., Edgett, K. S., 2006. Mars Orbiter Camera observations of Martian dust devils and their tracks (September 1997 to January 2006) and evaluation of theoretical vortex models. J. Geophys. Res. 111, E12002, doi:10.1029/2006JE002700.

Cardoso, J.-F., 1998. Blind signal separation: statistical principles. In: Proceedings of the IEEE. Special issue on blind identification and estimation. Vol. 9. pp. 2009-2025.

Cardoso, J.-F., 1999. High-order contrasts for independent component analysis. Neural Computation 11 (1), 157-192.

Chevrier, V., Poulet, F., Bibring, J.-P., 2007. Early geochemical environment of Mars as determined from thermodynamics of phyllosilicates. Nature 448, 
60-63, doi:10.1038/nature05961.

Clancy, R. T., Sandor, B. J., Moriarty-Schieven, G. H., Smith, M. D., 2006.

Mesospheric winds and temperatures from JCMT sub-millimeter CO line observations during the 2003 and 2005 Mars oppositions. In: Desjean et al. (2006).

Clancy, R. T., Wolff, M. J., Christensen, P. R., 2003. Mars aerosol studies with the MGS TES emission phase function observations: Optical depths, particles sizes, and ice cloud types versus latitude and solar longitude. Journal of Geophysical Research 108 (E9), 5098, doi:10.1029/2003JE002058.

Comon, P., 1990. Analyse en composantes indépendantes et identification aveugle. Traitement du Signal 7 (5), 435-450.

Comon, P., 1992. Independent component analysis. In: Lacoume, J. L. (Ed.), Higher-order statistics. Elsevier, Amsterdam, pp. 29-38.

Comon, P., 1994. Independent component analysis, a new concept? Signal processing 36, 287-314.

Desjean, M. C., Forget, F., Lopez-Valverde, M. (Eds.), 2006. Proceedings of the Second International Workshop on Mars atmosphere modelling and observations. Centre national d'etudes spatiales, European Space Agency, Instituto de astrofisica de Andalucia, Laboratoire de meteorologie dynamique / Centre national de recherches scientifiques, University of Oxford.

Erard, S., Drossart, P., Piccioni, G., 2008. Multivariate analysis of Virtis/venus-express night side and limb observations. J. Geophys. Res.Accepted for publication.

Fedorova, A. A., Lellouch, E., Titov, D. V., de Graauw, T., Feuchtgruber, H., 2002. Remote sounding of the martian dust from ISO spectroscopy in the $2.7 \mu \mathrm{m} \mathrm{CO}_{2}$ bands. Planetary and Space Science 50, 3-9.

Forget, F., 1998. Improved optical properties of the Martian atmospheric dust 
for radiative transfer calculations in the infrared. Geophys. Res. Lett. 25 (7), $1105-1108$.

Forget, F., Hourdin, F., Fournier, R., Hourdin, C., Talagrand, O., Collins, M.,

Lewis, S. R., Read, P. L., Huot, J.-P., 1999. Improved general circulation models of the martian atmosphere from the surface to above $80 \mathrm{~km}$. J. Geophys. Res. 104, 24155-24176.

Forget, F., Spiga, A., Dolla, B., Vinatier, S., Melchiorri, R., Drossart, P., Gendrin, A., Bibring, J.-P., Langevin, Y., Gondet, B., 2007. Remote sensing of surface pressure on Mars with the Mars Express/OMEGA spectrometer: 1. Retrieval method. J. Geophys. Res. 112, E08S15, doi:10.1029/2006JE002871.

Formisano, V., Angrilli, F., Arnold, G., Atreya, S., Bianchini, G., Biondi, D., Blanco, A., Bleka, M. I., Coradini, A., Colangeli, L., Ekonomov, A., Encrenaz, T., Esposito, F., Fonti, S., Giuranna, M., Grassi, D., Gnedykh, V., Grigoriev, A., Hansen, G., Hirsh, H., Khatuntsev, I., Kiselev, A., Ignatiev, N., Jurewicz, A., Lellouch, E., Moreno, J. L., Marten, A., Mattana, A., Maturilli, A., Mencarelli, E., Michalska, M., Moroz, V., Moshkin, B., Nespoli, F., Nikolsky, Y., Orfei, R., Orleanski, P., Orofino, V., Palomba, E., Patsaev, D., Piccioni, G., Rataj, M., Rodrigo, R., Rodriguez, J., Rossi, M., Saggin, B., Titov, D., Zasova, L., 2005. The Planetary Fourier Spectrometer (PFS) onboard the European Mars Express mission. Planetary and Space Science $53,963-974$.

Forni, O., Poulet, F., Bibring, J.-P., Erard, S., Gomez, C., Langevin, Y., Gondet, B., OMEGA team, 2005. Component separation of OMEGA spectra with ICA. In: 36th Annual Lunar and Planetary Science Conference. Vol. 63. p. 1623.

Fouchet, T., Lellouch, E., Ignatiev, N. I., Forget, F., Titov, D. V., Tschimmel, 
M., Montmessin, F., Formisano, V., Giuranna, M., Maturilli, A., Encrenaz, T., 2007. Martian water vapor: Mars Express PFS/LW observations. Icarus 190, 32-49.

Gierasch, P. J., Goody, R. M., 1972. The effect of dust on the temperature of the Martian atmosphere. J. Atmos. Sci. 29 (2), 400-402.

Giuranna, M., Formisano, V., Biondi, D., Ekonomov, A., Fonti, S., Grassi, D., Hirsch, H., Khatuntsev, I., Ignatiev, N., Malgoska, M., Mattana, A., Maturilli, A., Mencarelli, E., Nespoli, F., Orfei, R., Orleanski, P., Piccioni, G., Rataj, M., Saggin, B., Zasova, L., 2005. Calibration of the Planetary Fourier Spectrometer long wavelength channel. Planet. Space Sci. 53 (10), 993-1007, doi:10.1016/j.pss.2005.02.007.

Gomez, C., Borgne, H. L., Allemand, P., Delacourt, C., Ledru, P., 2007. Nfindr method versus independent component analysis for lithological identification in hyperspectral imagery. International Journal of Remote Sensing $28(23), 5315-5338$.

Hanel, R. A., Conrath, B. J., Hovis, W. A., Kunde, V. G., Lowman, P. D.,

Pearl, J. C., Prabhakara, C., Schlachman, B., Levin, G. V., 1972. Infrared Spectroscopy Experiment and the Mariner 9 mission: Preliminary results. Science 175, 305-308.

Hyvärinen, A., Karhunen, J., Oja, E., 2001. Independent Component Analysis. John Wiley, New York.

Jacquinet-Husson, N., Arié, E., Ballard, J., Barbe, A., Bjoraker, G., Bonnet, B., Brown, L. R., Camy-Peyret, C., Champion, J. P., Chédin, A., Chursin, A., Clerbaux, C., Duxbury, G., Flaud, J. M., Fourrié, N., Fayt, A., Graner, G., Gamache, R., Goldman, A., Golovko, V., Guelachvili, G., Hartmann, J. M., Hillico, J. C., Hillman, J., Lefevre, G., Lellouch, E., Mikhailenko, S. N., Naumenko, O. V., Nemtchinov, V., Newnham, D. A., Nikitin, A., 
Orphal, J., Perrin, A., Reuter, D. C., Rinsland, C. P., Rosenmann, L., Rothman, L. S., Scott, N. A., Selby, J., Sinitsa, L. N., Sirota, J. M., Smith, A. M., Smith, K. M., Tyuterev, V. G., Tipping, R. H., Urban, S., Varanasi, P., Weber, M., 1999. The 1997 spectroscopic geisa databank. J. Quant. Spectrosc. Radiat. Transfer 62, 205-254.

Johnson, J. R., Grundy, W. M., Lemmon, M. T., 2003. Dust deposition at the mars pathfinder landing site: observations and modeling of visible/nearinfrared spectra. Icarus 163, 330-346.

Kahn, R. A., Martin, T. Z., Zurek, R. W., 1992. The Martian dust cycle. In: Kieffer, H. H., Jakosky, B. M., Snyder, C. W., Matthews, M. S. (Eds.), Mars. University of Arizona Press, pp. 1017-1053.

Kahre, M. A., Hollingsworth, J. L., Haberle, R. M., Murphy, J. M., 2008. Investigations of the variability of dust particle sizes in the martian atmosphere using the NASA Ames General Circulation Model. Icarus 195, 576-597, doi:10.1016/j.icarus.2008.01.023.

Kahre, M. A., Murphy, J. R., Haberle, R. M., 2006. Modeling the Martian dust cycle and surface dust reservoirs with the NASA Ames general circulation model. J. Geophys. Res. 111 (E06008), doi:10.1029/2005JE002588.

Kahre, M. A., Murphy, J. R., Haberle, R. M., Montmessin, F., Schaeffer, J., 2005. Simulating the Martian dust cycle with a finite surface dust reservoir. Geophys. Res. Lett. 32, L20204, doi:10.1029/2005GL023495.

Lewis, S. R., Collins, M., Read, P. L., Forget, F., Hourdin, F., Fournier, R., Hourdin, C., Talagrand, O., Huot, J.-P., 1999. A climate database for Mars. Journal of Geophysical Research 104 (E10), 24177-24194.

Markiewicz, W. J., Sablotny, R. M., Keller, H. U., Thomas, N., Titov, D., Smith, P. H., 1999. Optical properties of the martian aerosols as derived from imager for mars pathfinder midday sky brightness data. J. Geophys. 
Res 104, 9009-9018.

Mateshvili, N., Fussen, D., Vanhellemont, F., Bingen, C., Dodion, J., Montmessin, F., Perrier, S., Dimarellis, E., Bertaux, J.-L., 2007. Martian ice cloud distribution obtained from SPICAM nadir UV measurements. Journal of Geophysical Research 112, E07004, doi:10.1029/2006JE002827.

Meador, W. E., Weaver, W. R., 1980. Two-stream approximations to radiative transfer in planetary atmospheres: A unified description of existing methods and a new improvement. J. Atmos. Sci. 37, 630-643.

Michaels, T. I., Rafkin, S. C. R., 2004. Large-eddy simulations of atmospheric convection on Mars. Q. J. R. Meteorol. Soc. 130, 1251-1274, doi:10.1256/qj.02.169.

Michelangeli, D. V., Toon, O. B., Haberle, R. M., Pollack, J. B., 1993. Numerical simulations of the formation and evolution of water ice clouds in the Martian atmosphere. Icarus 100, 261-285.

Moreno, R., Lellouch, E., Encrenaz, T., Forget, F., Chassefiere, E., Hourdin, F., Guilloteau, S., 2006. Wind measurements in Mars' middle atmosphere at equinox and solstice: IRAM Plateau de Bure interferometric CO observations. In: Desjean et al. (2006).

Murphy, J. R., Pollack, J. B., Haberle, R. M., Leovy, C. B., Toon, O. B., Schaeffer, J., 1995. Three-dimensional numerical simulation of Martian dust storms. J. Geophys. Res. 100, 26357-26376.

Newman, C. E., Lewis, S. R., Read, P. L., Forget, F., 2002a. Modeling the Martian dust cycle, 1: Representations of dust transport processes. Journal of Geophysical Research 107 (E12), 5123.

Newman, C. E., Lewis, S. R., Read, P. L., Forget, F., 2002b. Modeling the Martian dust cycle, 2: Multiannual radiatively active dust transport simulations. Journal of Geophysical Research 107 (E12), 5124. 
Ockert-Bell, M. E., Bell, III, J. F., Pollack, J. B., McKay, C. P., Forget, F., 1997. Absorption and scattering properties of the Martian dust in the solar wavelengths. J. Geophys. Res. 102 (E4), 9039-9050.

Pollack, J. B., Colburn, D. S., Flaser, M., Kahn, R., Carlston, C. E., Pidek, D., Jun. 1979. Properties and effects of dust particles suspended in the Martian atmosphere. Journal of Geophysical Research 84 (B6), 2929-2945.

Poulet, F., Bibring, J.-P., Mustard, J. F., Gendrin, A., Mangold, N., Langevin, Y., Arvidson, R. E., Gondet, B., Gomez, C., 2005. Phyllosilicates on Mars and implications for early martian climate. Nature 438, 623-627, doi:10.1038/nature04274.

Rodin, A. V., Clancy, R. T., Wilson, R. J., 1999. Dynamical properties of Mars water ice clouds and their interactions with atmospheric dust and radiation. Adv. Space Res. 23 (9), 1577-1585.

Schmidt, F., 2007. Classification de la surface de mars par imagerie spectrale omega. suivi spatio-temporel et étude des dépôts saisonniers de $\mathrm{co}_{2}$ et $\mathrm{h}_{2} \mathrm{O}$. Ph.D. thesis, Université Joseph Fourier, Grenoble.

Smith, M. D., 2002. The annual cycle of water vapor on Mars as observed by the Thermal Emission Spectrometer. Journal of Geophysical Research 107 (E11), 5115.

Smith, M. D., 2004. Interannual variability in TES atmospheric observations in Mars during 1999-2003. Icarus 167, 148-165, doi:10.1016/j.icarus.2003.09.010.

Smith, M. D., Bandfield, J. L., Christensen, P. R., 2000. Separation of atmospheric and surface spectral features in Mars Global Surveyor Thermal Emission Spectrometer (TES) spectra. J. Geophys. Res. 105, 9589-9608.

Smith, M. D., Conrath, B. J., Pearl, J. C., Christensen, P. R., 2002. NOTE: Thermal Emission Spectrometer observations of Martian planet-encircling 
dust storm 2001A. Icarus 157, 259-263.

Spiga, A., Forget, F., Dolla, B., Vinatier, S., Melchiorri, R., Drossart, P., Gendrin, A., Bibring, J.-P., Langevin, Y., Gondet, B., 2007. Remote sensing of surface pressure on Mars with the Mars Express/OMEGA spectrometer: 2. Meteorological maps. J. Geophys. Res. 112, E08S16, doi:10.1029/2006JE002870.

Tegen, I., Lacis, A. A., 1996. Modeling of particle size distribution and its influence on the radiative properties of mineral dust aerosol. J. Geophys. Res. 101 (D14), 19237-19244.

Titov, D. V., Fedorova, A. A., Haus, R., 2000. A new method of remote sounding of the martian aerosols by means of spectroscopy in the $2.7 \mu \mathrm{m}$ band. Planet. Space Sci. 48, 67-74.

Toigo, A. D., Richardson, M. I., Wilson, R. J., Wang, H., Ingersoll, A. P., 2002. A first look at dust lifting and dust storms near the south pole of Mars with a mesoscale model. Journal of Geophysical Research 107 (E7).

Tomasko, M. G., Loose, L. R., Lemmon, M., Smith, P. H., Wegryn, E., 1999. Properties of dust in the Martian atmosphere from the Imager of Mars Pathfinder. Journal of Geophysical Research 104 (E4), 8987-9007.

Toon, O. B., McKay, C. P., Ackerman, T. P., Santhanam, K., 1989. Rapid calculation of radiative heating rates and photodissociation rates in inhomogeneous multiple scattering atmosphere. J. Geophys. Res. 94, 16287-16301.

Tran, T. T., Rannou, P., 2004. Comparing 3D spherical Monte-Carlo and 2stream parallel plane simulation of far-field backscattering image of Titan. Notes du Pôle de Planétologie de l'Institut Pierre Simon Laplace (2), 18.

Tyler, Jr., D., Barnes, J. R., Skyllingstad, E. D., 2008. Mesoscale and largeeddy simulation model studies of the Martian atmosphere in support of Phoenix. J. Geophys. Res. 113, E00A12, doi:10.1029/2007JE003012. 
Vincendon, M., Langevin, Y., Poulet, F., Bibring, J.-P., Gondet, B., 2007.

Recovery of surface reflectance spectra and evaluation of the optical depth of aerosols in the near-ir using a Monte Carlo approach: Application to the OMEGA observations of high-latitude regions of Mars. J. Geophys. Res. 112, E08S13, doi:10.1029/2006JE002845.

Wolff, M. J., Clancy, R. T., 2003. Constraints of the size of Martian aerosols from Thermal Emission Spectrometer observations. J. Geophys. Res. 108 (E9), 5097, doi:10.1029/2003JE002057.

Wolff, M. J., Smith, M. D., Clancy, R. T., Spanovich, N., Whitney, B. A., Lemmon, M. T., Bandfield, J. L., Banfield, D., Ghosh, A., Landis, G., Christensen, P. R., Bell, J. F., Squyres, S. W., Dec. 2006. Constraints on dust aerosols from the Mars Exploration Rovers using MGS overflights and MiniTES. J. Geophys. Res. 111 (E10), 12. 
Table 1

Errors for 3 values of the retrieved dust optical thickness $\tau$. The errors caused by uncertainties in $\omega_{0}$ and in the surface albedo are given separately and summed at the last line.

\begin{tabular}{|c|c|c|c|}
\hline Errors at & $\tau=4$ & $\tau=6$ & $\tau=8$ \\
\hline$\omega_{0}$ & ${ }_{-0.9}^{+0.5}$ & ${ }_{-0.9}^{+0.5}$ & ${ }_{-0.7}^{+0.5}$ \\
\hline Surface albedo & \pm 0.2 & \pm 0.1 & \pm 0.1 \\
\hline Total error & ${ }_{-0.92}^{+0.54}$ & ${ }_{-0.91}^{+0.51}$ & ${ }_{-0.71}^{+0.51}$ \\
\hline
\end{tabular}


Fig. 1. Tracks of orbit\#1201 session 3 and orbit\#1212 session 3 mapped on cylindrical projection of the Martian surface. The dust storm studied in this paper was seen on orbit \#1212 between the latitudes shown by the dashed lines. The PFS FOV of orbit \#1212 for the temperature profile inversion is shown with the oval. The local time of observations was about 13.30 .

Fig. 2. Comparison of the OMEGA spectra averaged over the pixels in the range $2^{\circ}$ $\mathrm{S}-4^{\circ} \mathrm{S}$ common to orbit\#1201 (dotted line) and orbit\#1212 (solid line).

Fig. 3. Comparison of the PFS spectra averaged over the range $2^{\circ} \mathrm{S}-4^{\circ} \mathrm{S}$ obtained during orbit\#1201 (dotted line) and orbit\#1212 (solid line).

Fig. 4. Spatial maps of the two major components of the ICA decomposition of orbit \#1212. Left panel: the dust component. Right panel: the surface aldedo component.

Fig. 5. The behavior of the single-scattering albedo $\omega_{0}$ with 3 different values of dust optical thickness $\tau=5,10,20$. The value $\tau=10$ with the flattest behavior of $\omega_{0}$ was used for the retrieval.

Fig. 6. Left: The retrieved single-scattering albedo (solid line) and comparison with previous studies of Fedorova et al. (2002) (dashed line), Ockert-Bell et al. (1997) (stars), and Tomasko et al. (1999) (crosses). Right: Contours of the I/F as a function of single-scattering albedo $\omega_{0}$ and asymmetry parameter $g$ for the infinite optical depth case. The star represents the $\omega_{0}$ derived from our OMEGA data at $1.07 \mu \mathrm{m}$. The error bar shows the uncertainty on $\omega_{0}$ due to the $15 \%$ uncertainty on OMEGA absolute calibration.

Fig. 7. The dust optical thickness at $1.0 \mu \mathrm{m}$ in the area of the storm $\left(0.4-4.7^{\circ} \mathrm{S}\right)$. The color scale ranges between 0-9.9. 
Fig. 8. The latitude-pressure cross-sections of the temperature structure inverted from PFS/LW observations for orbit \#1201 (top) and \#1212 (bottom). The positions of the temperature profiles of Fig. ?? are marked with lines, styles match those of Fig. ??: the solid line is from the dusty orbit 1212 inside the dust storm, the dashed line from the dustfree orbit 1201 at the storm area, and the dash-dotted line is from the dusty orbit outside the dust storm. The local time of observations at the dust storm region was 13.30 .

Fig. 9. The temperature profiles retrieved from PFS data: the solid line is from the dusty orbit 1212 inside the dust storm, the dashed line from the dustfree orbit 1201 at the storm area, and the dash-dotted line is from the dusty orbit outside the dust storm. The local time of observations was 13.30 .

Fig. 10. The analysed PFS temperature profile (the solid line) and 1-D model temperature profiles with different dust vertical profiles. The LMD 1-D model $\tau=4.23$ results: the short-dashed line is the profile with well-mixed dust up to $55 \mathrm{~km}$. The profiles with limited dust vertical extent are: dotted line with dust storm top at $10 \mathrm{~km}$, long-dashed line $15 \mathrm{~km}$, dash-three-dot line $25 \mathrm{~km}$, and dash-dot line $35 \mathrm{~km}$. In all the profiles the well-mixed background dust optical thickness is $\tau=0.2$. The local time of the observation and the model profiles is 13.30 and the model results are from sol 10. 
Fig. 11. Temperature profiles from the first sol of the model run with $\tau=4.23$ and dust top altitude $55 \mathrm{~km}$ at local times $00,08,13$ and 20 . The model was started at 00 LT and initialised with an isothermal temperature profile. 


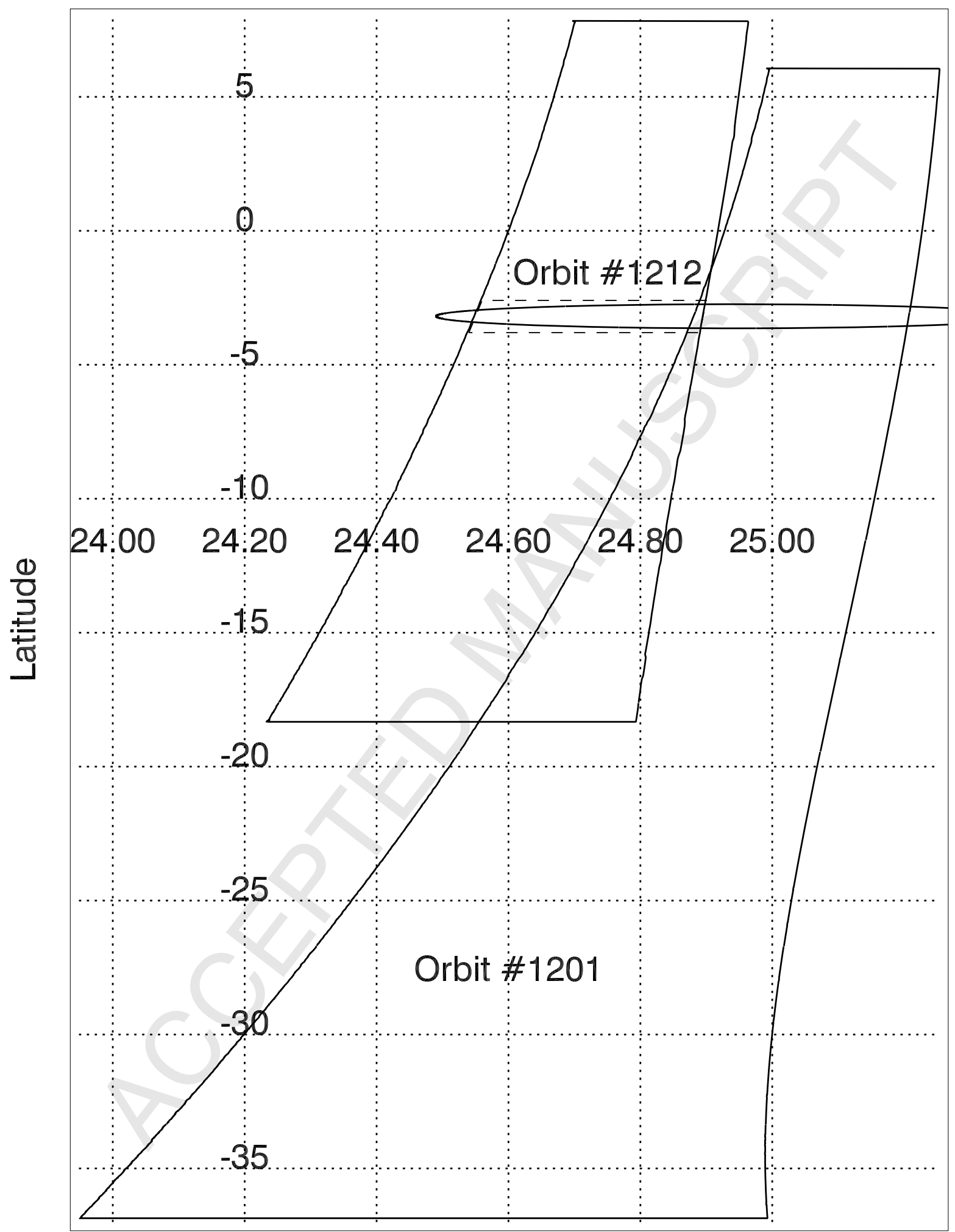

\section{Longitude}


Reflectance (I/F)

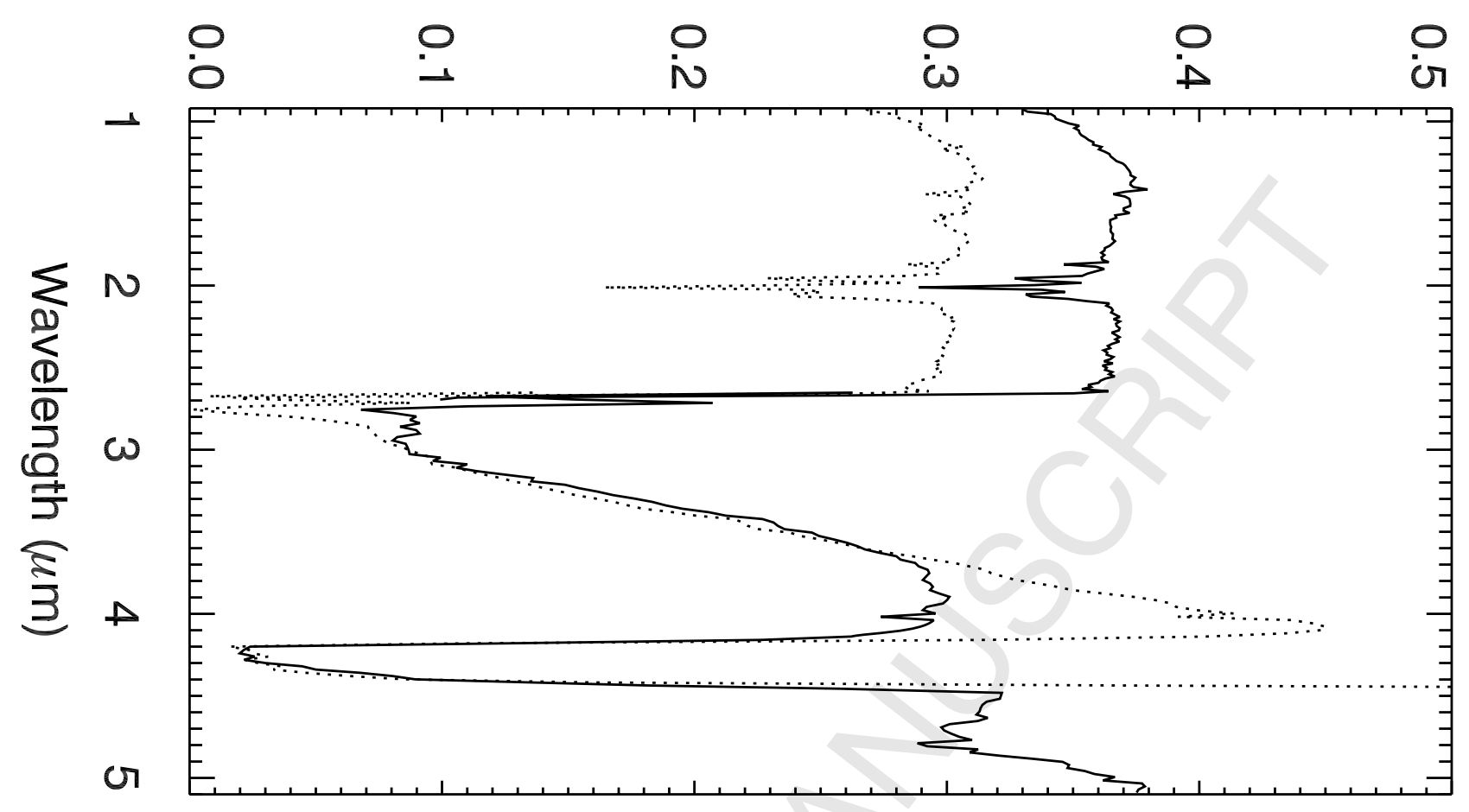

Brightness Temperature (K)

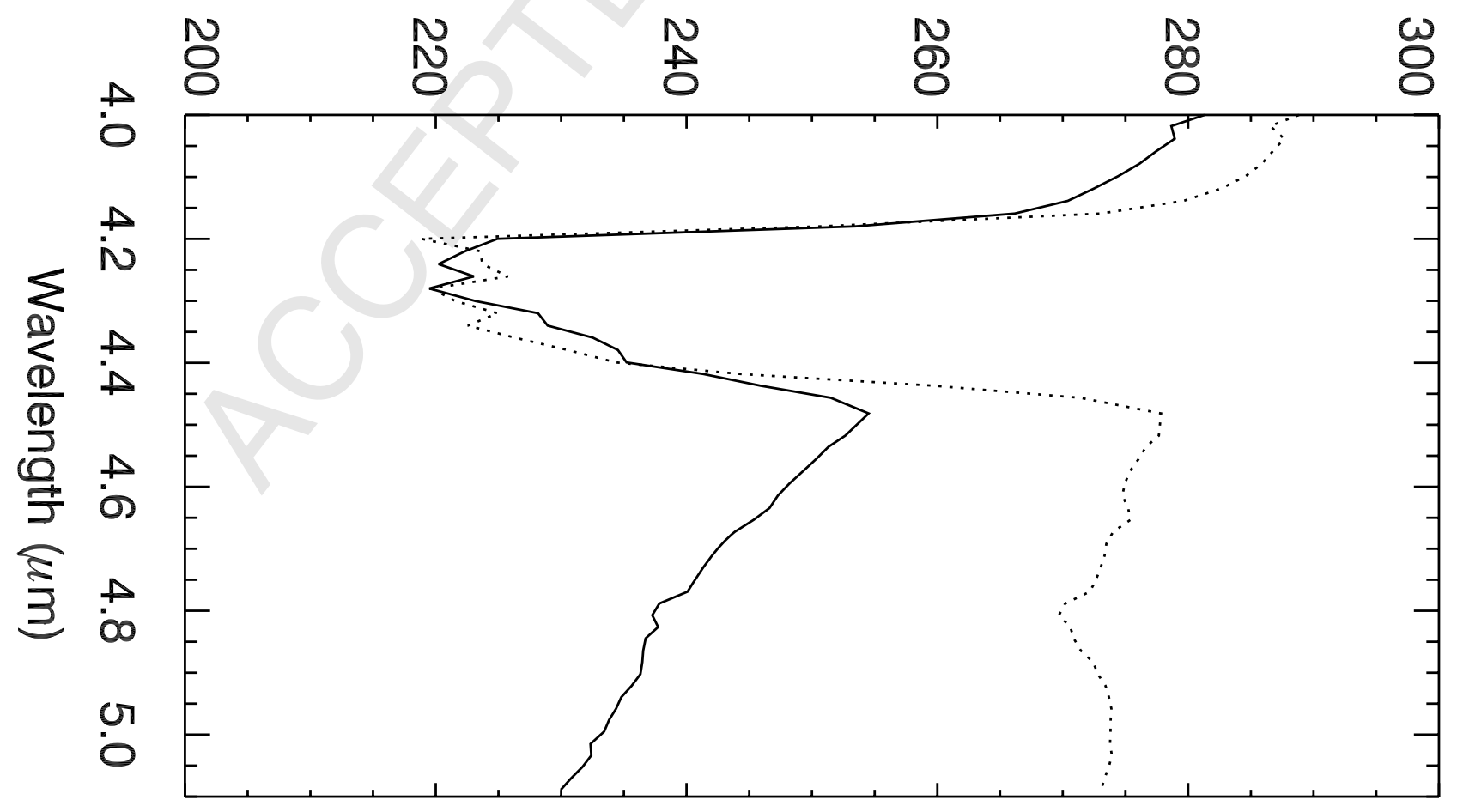




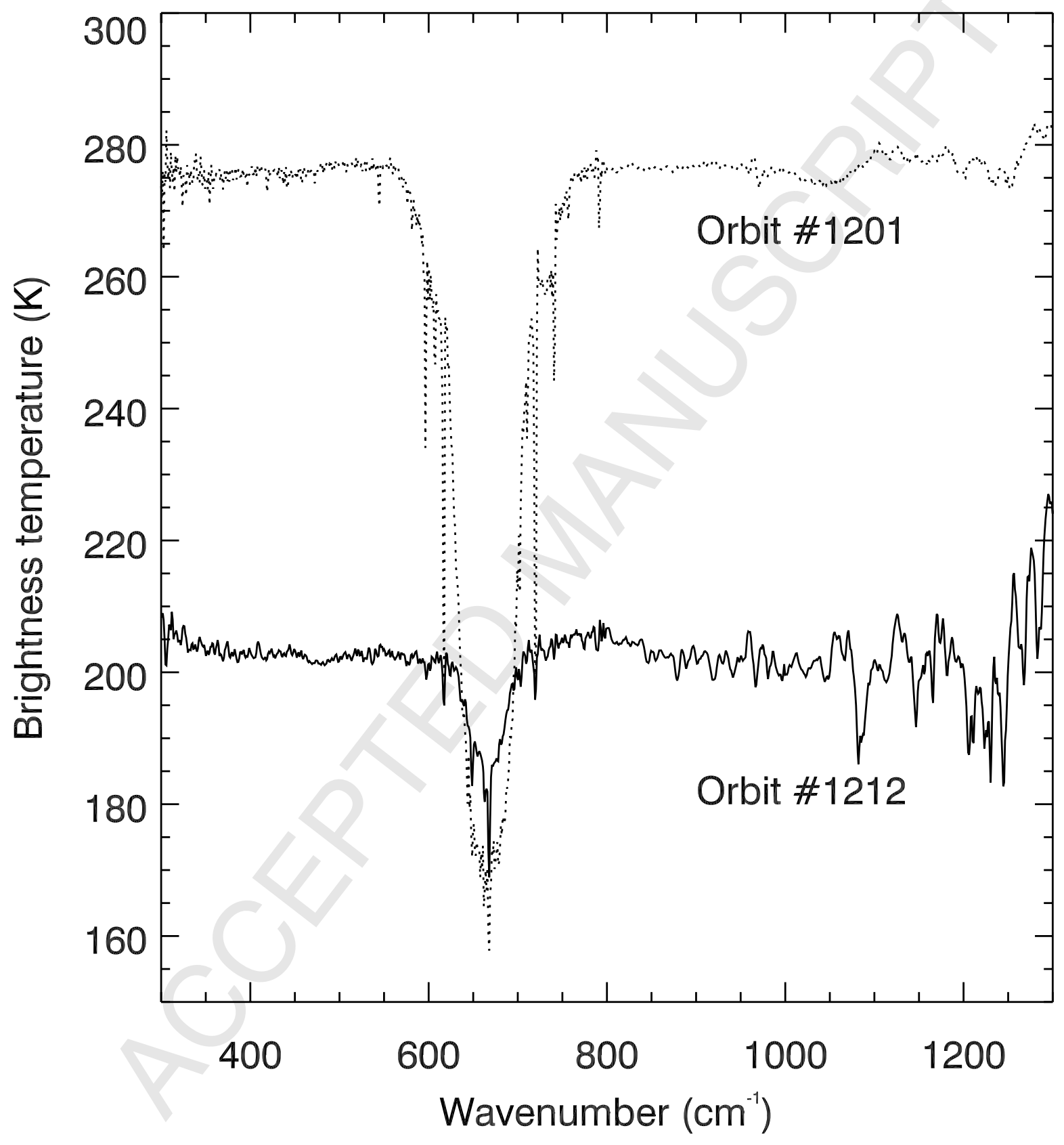




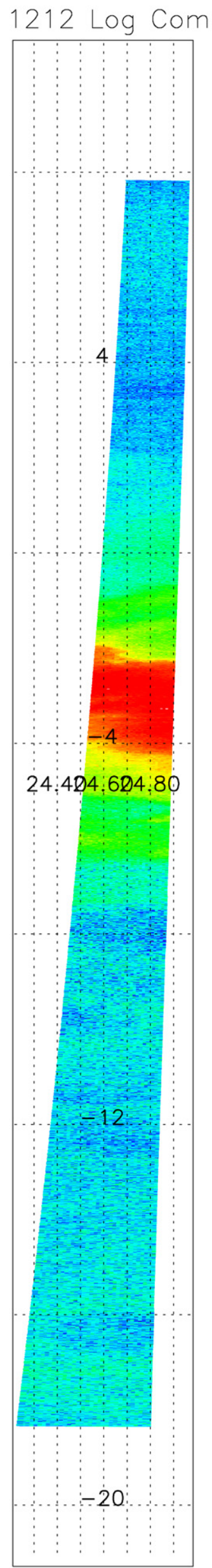




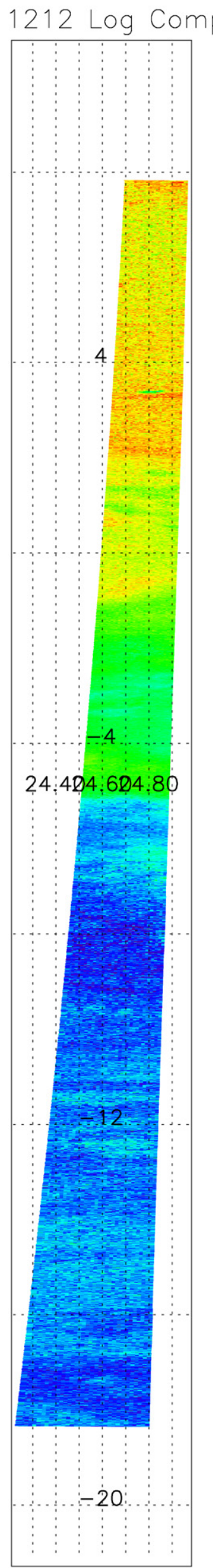




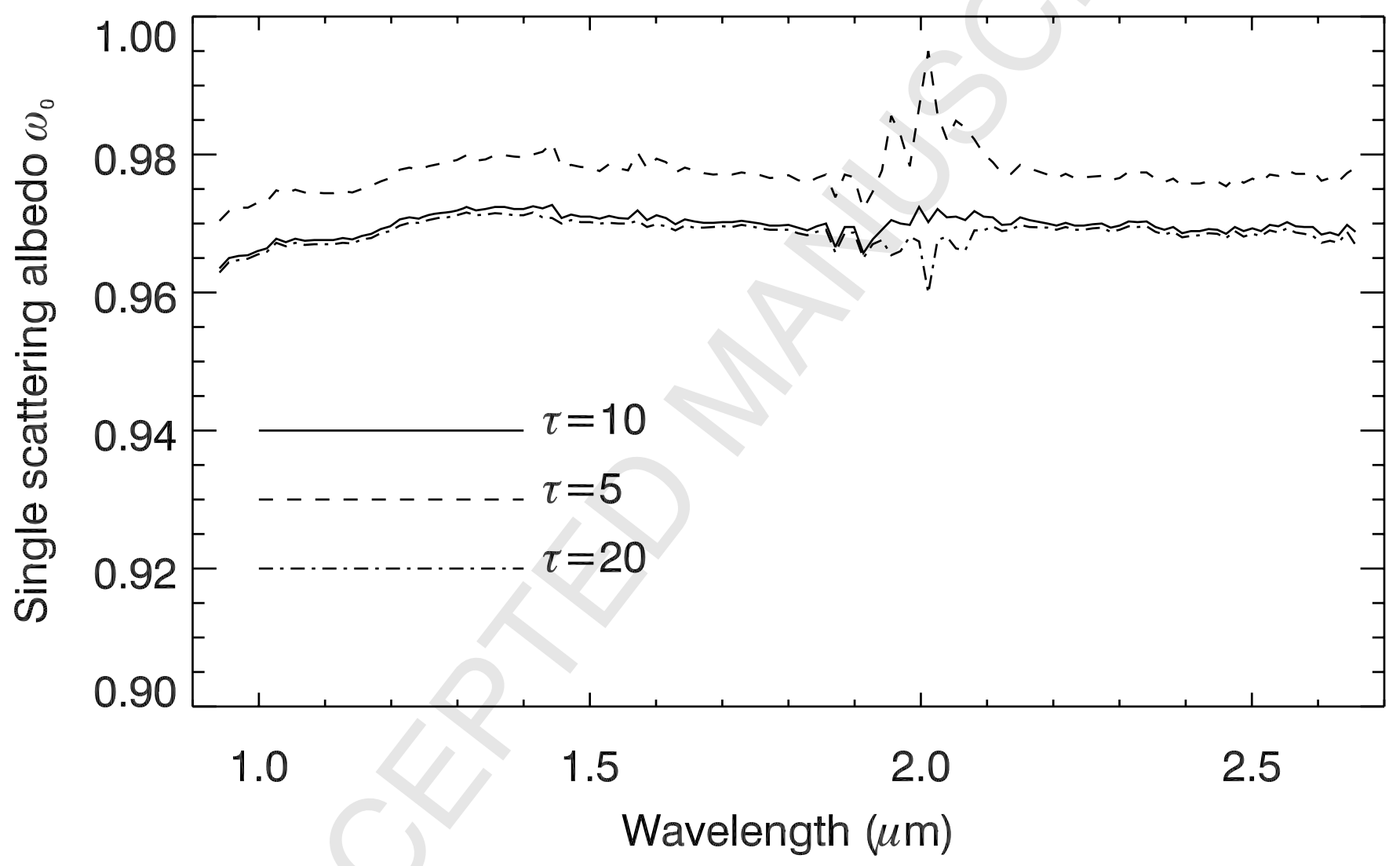




\section{ACCEPTED MANUSCRIP}

Single scattering albedo $\omega_{0}$

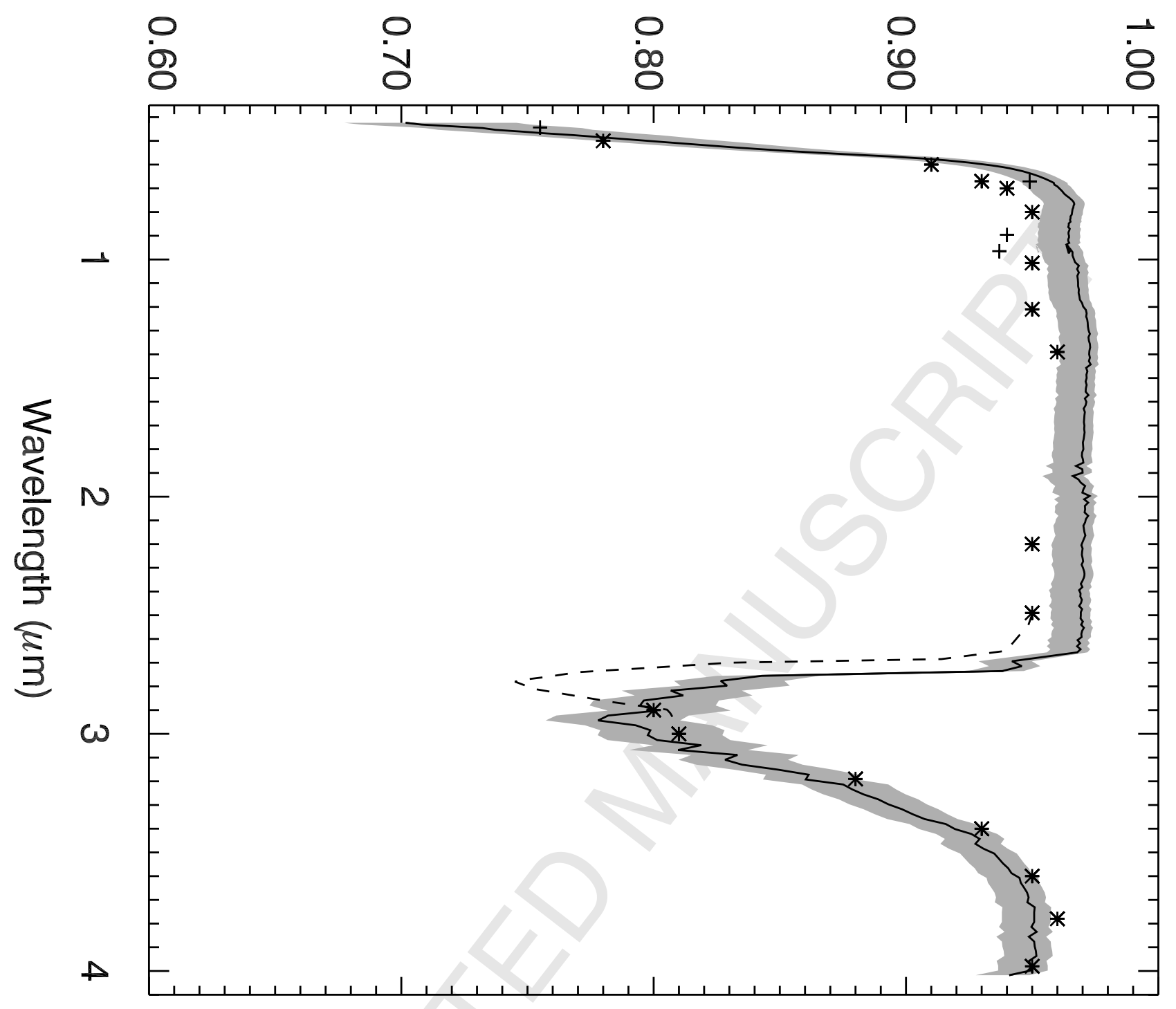

Asymmetry parameter $\mathrm{g}$

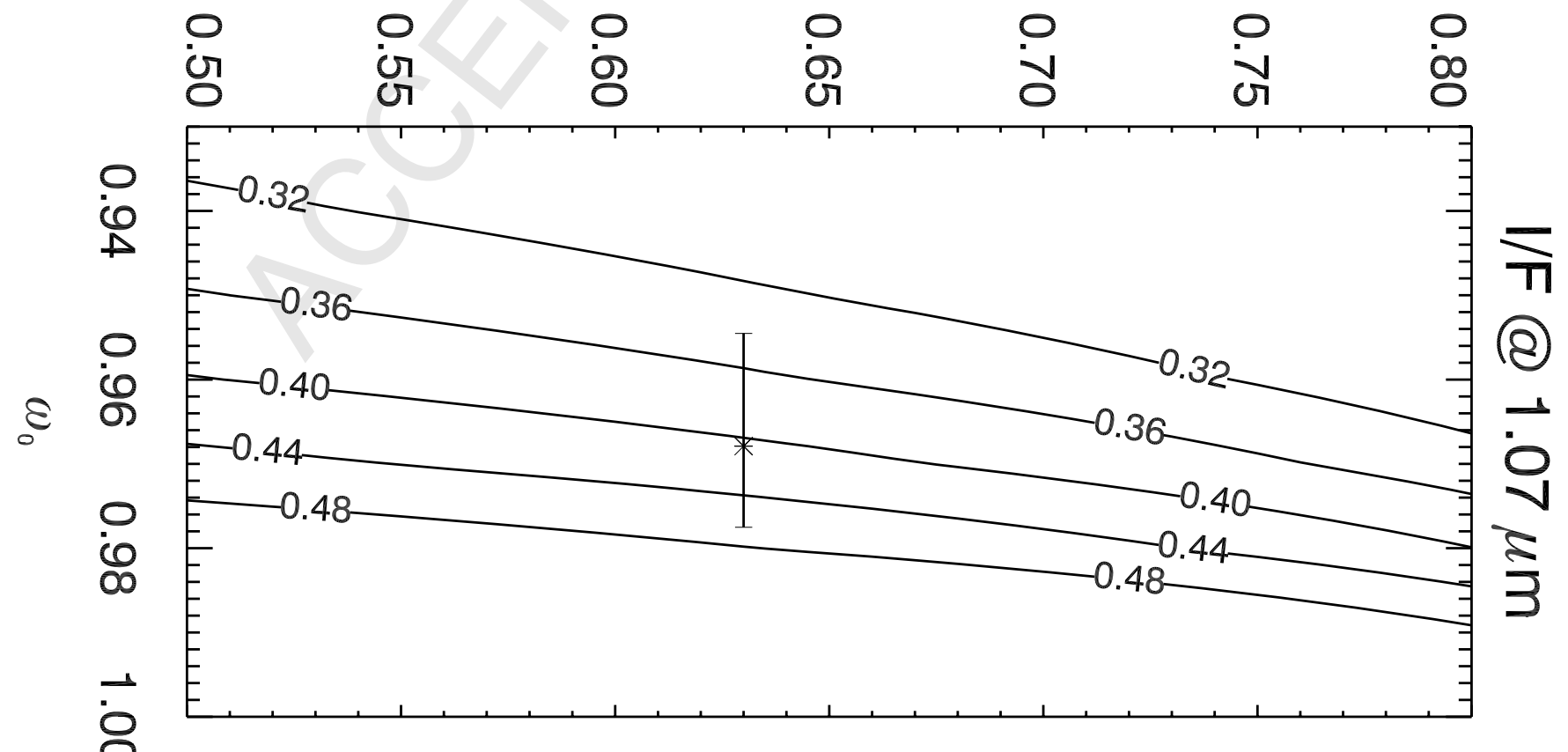




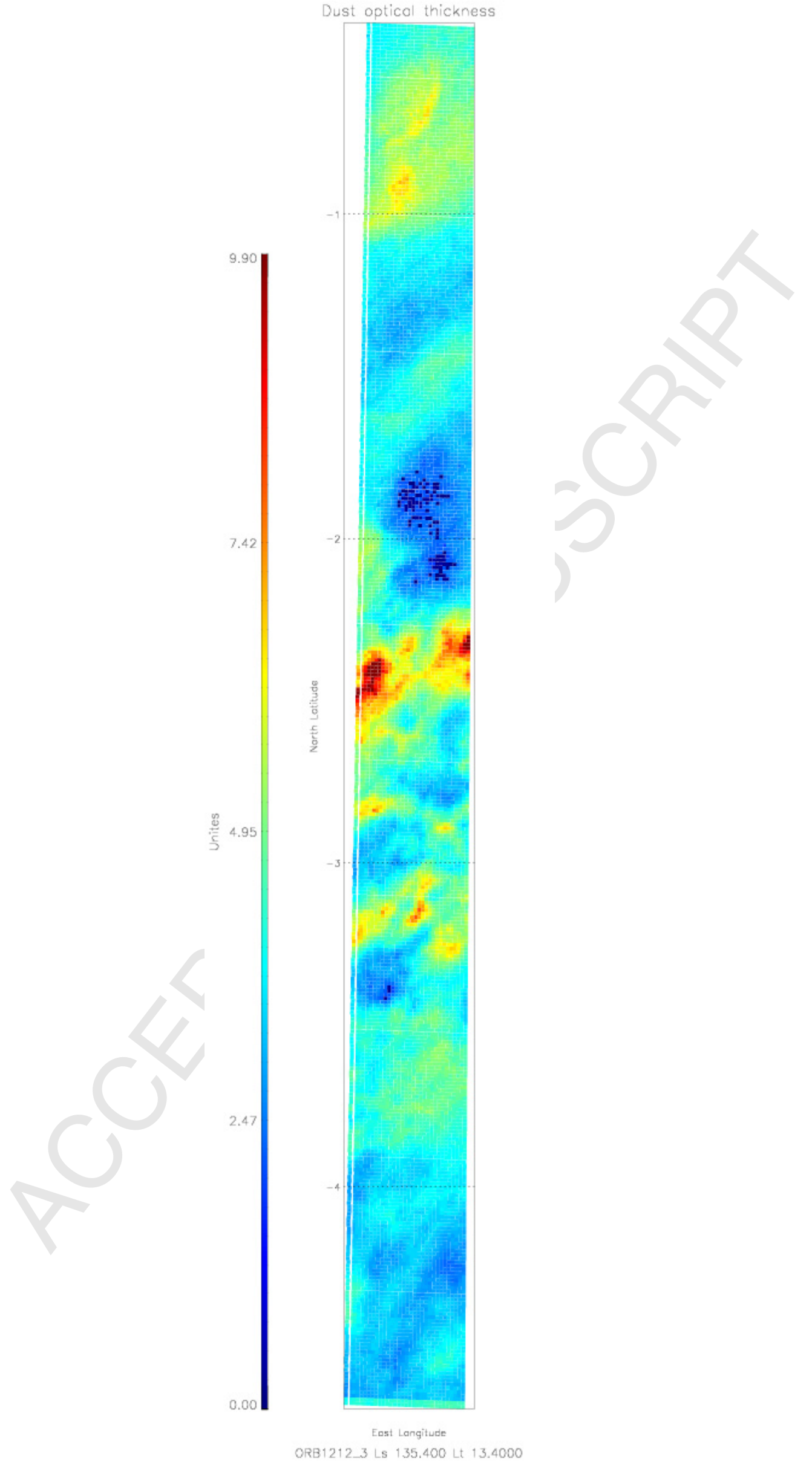




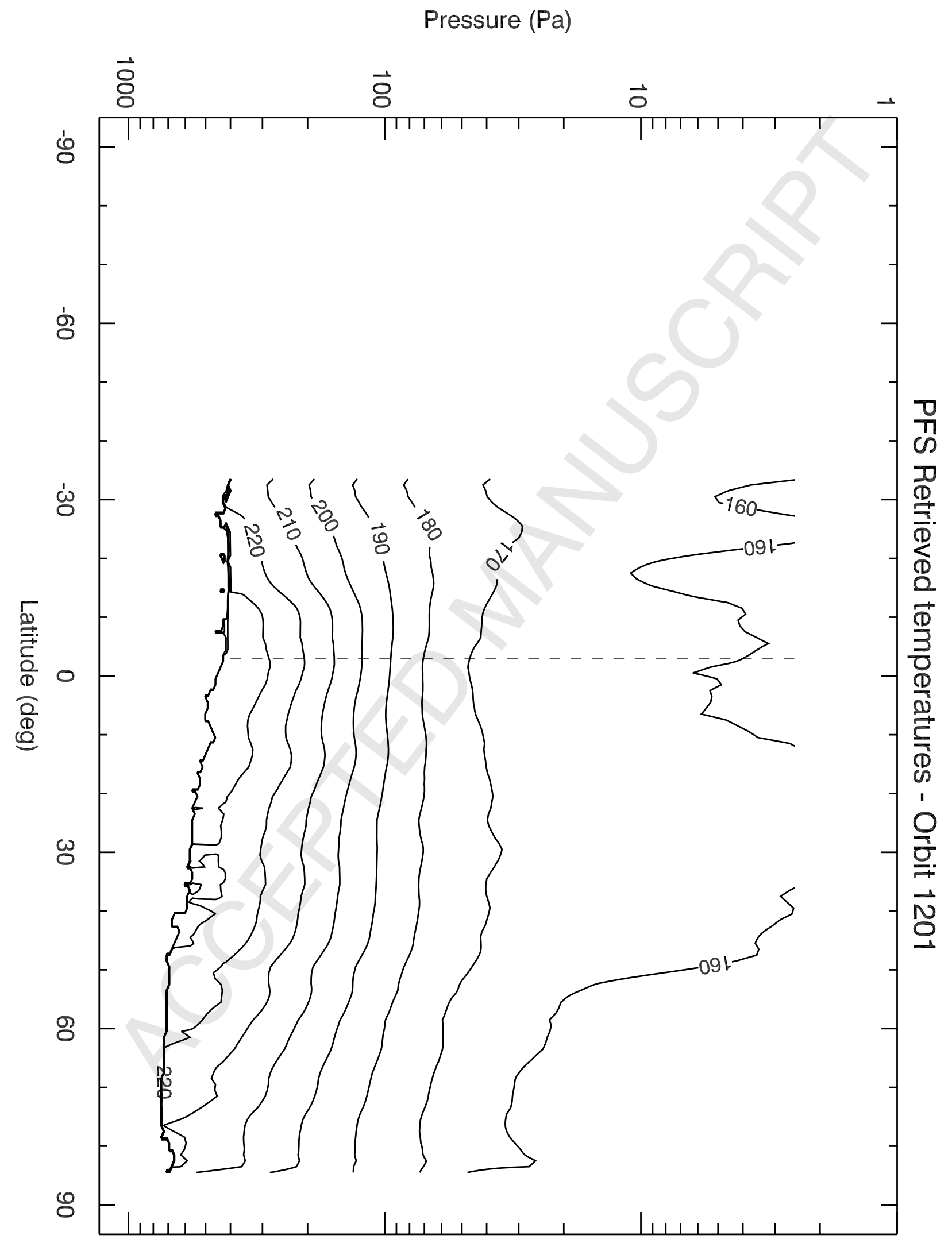




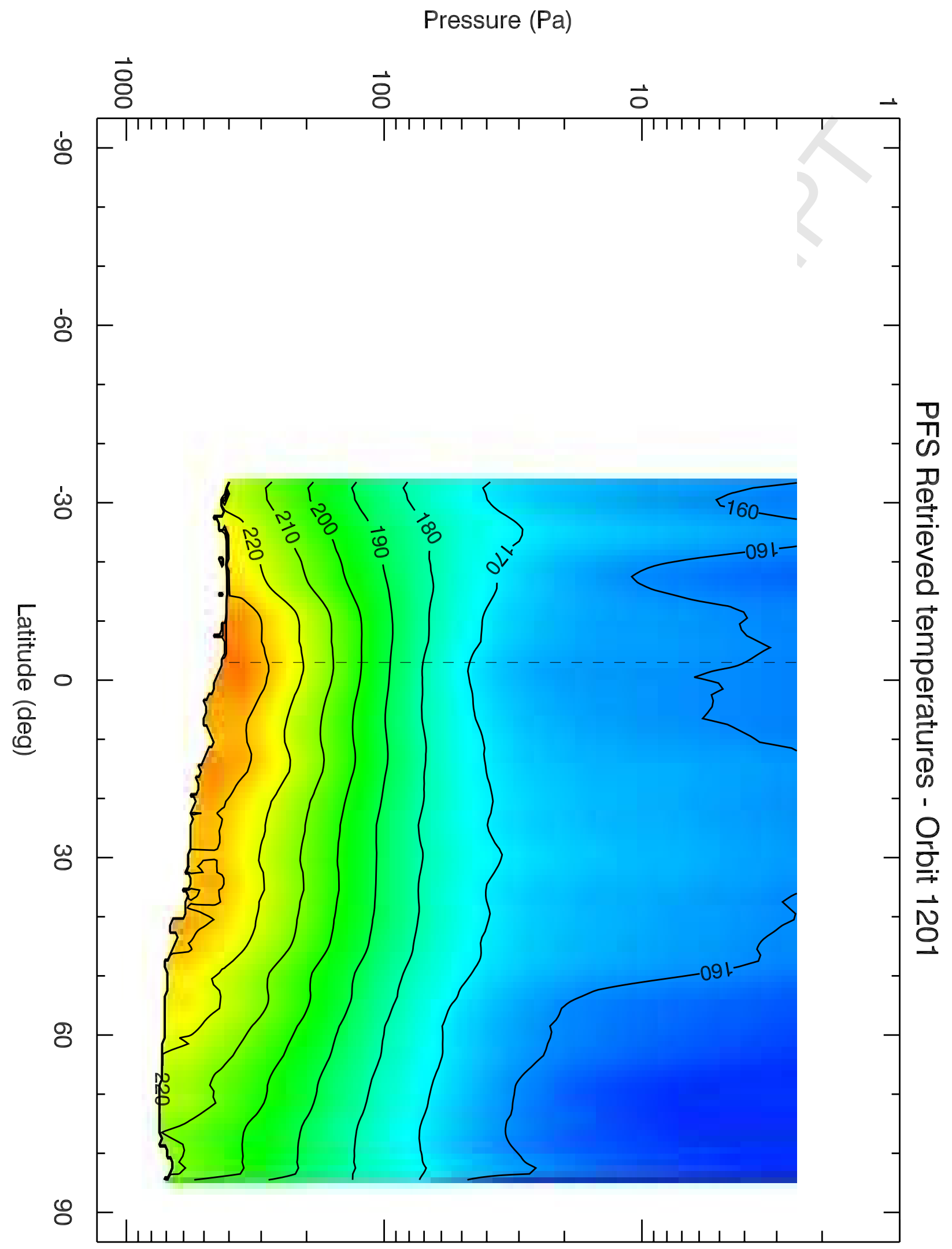




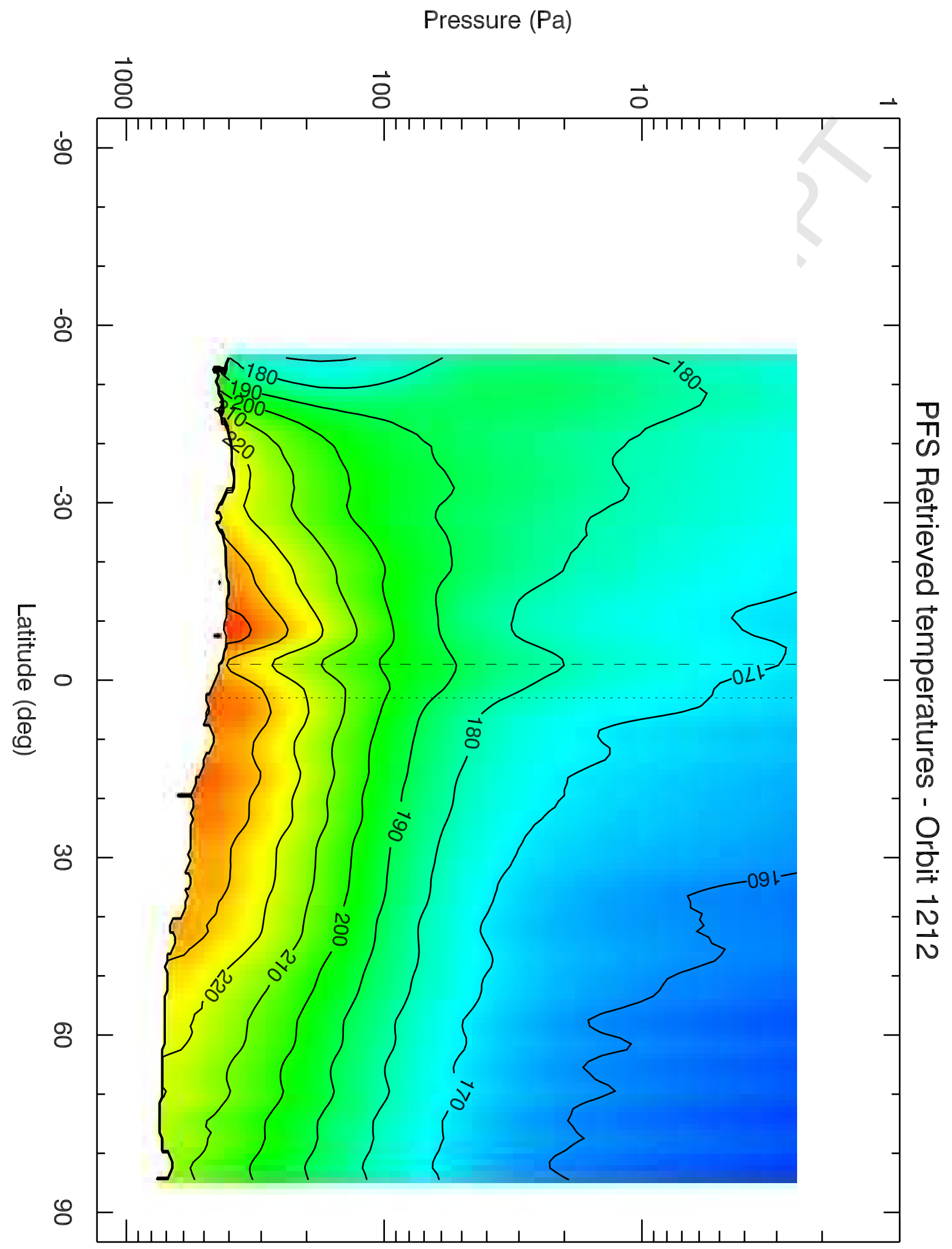




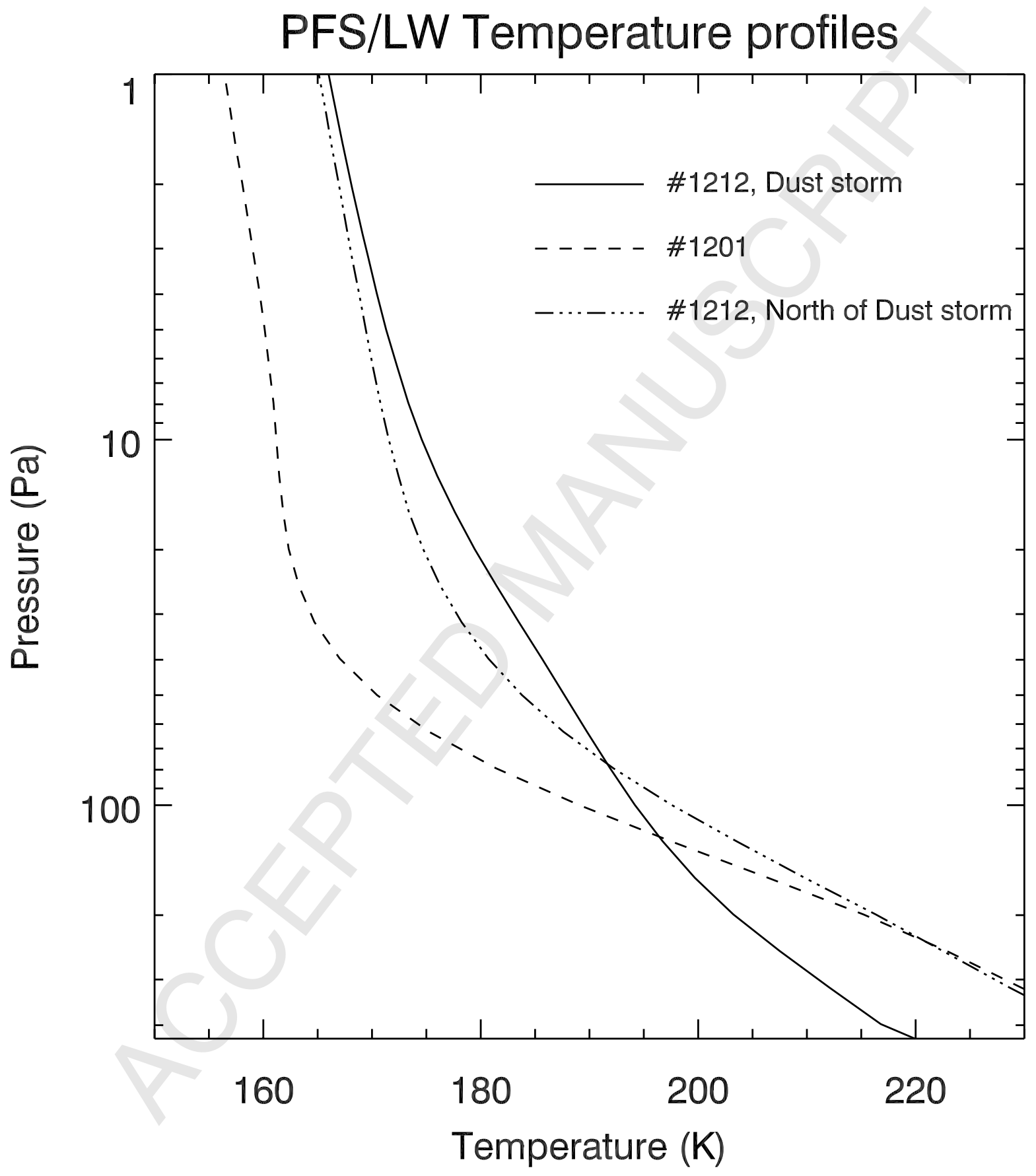




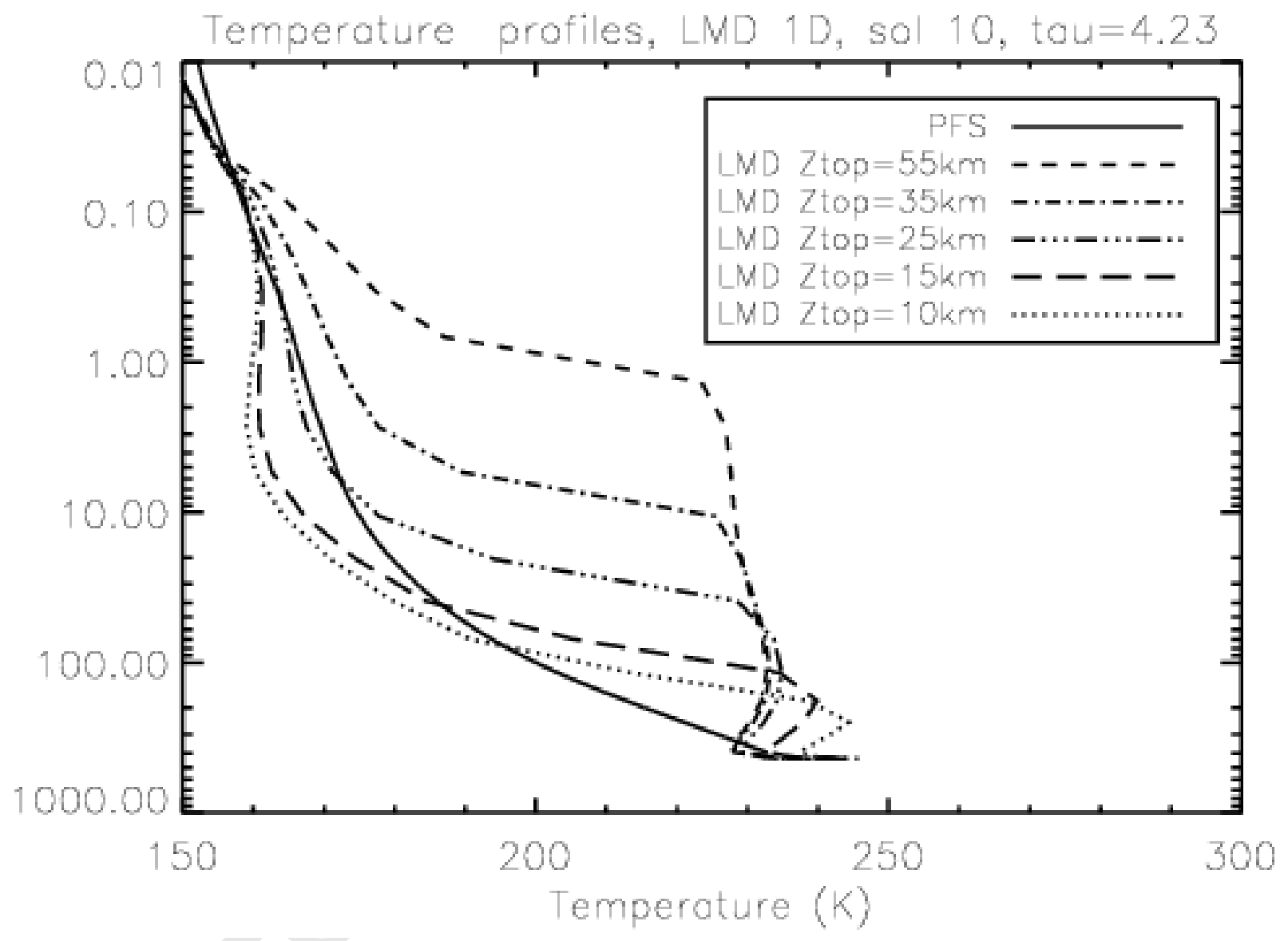




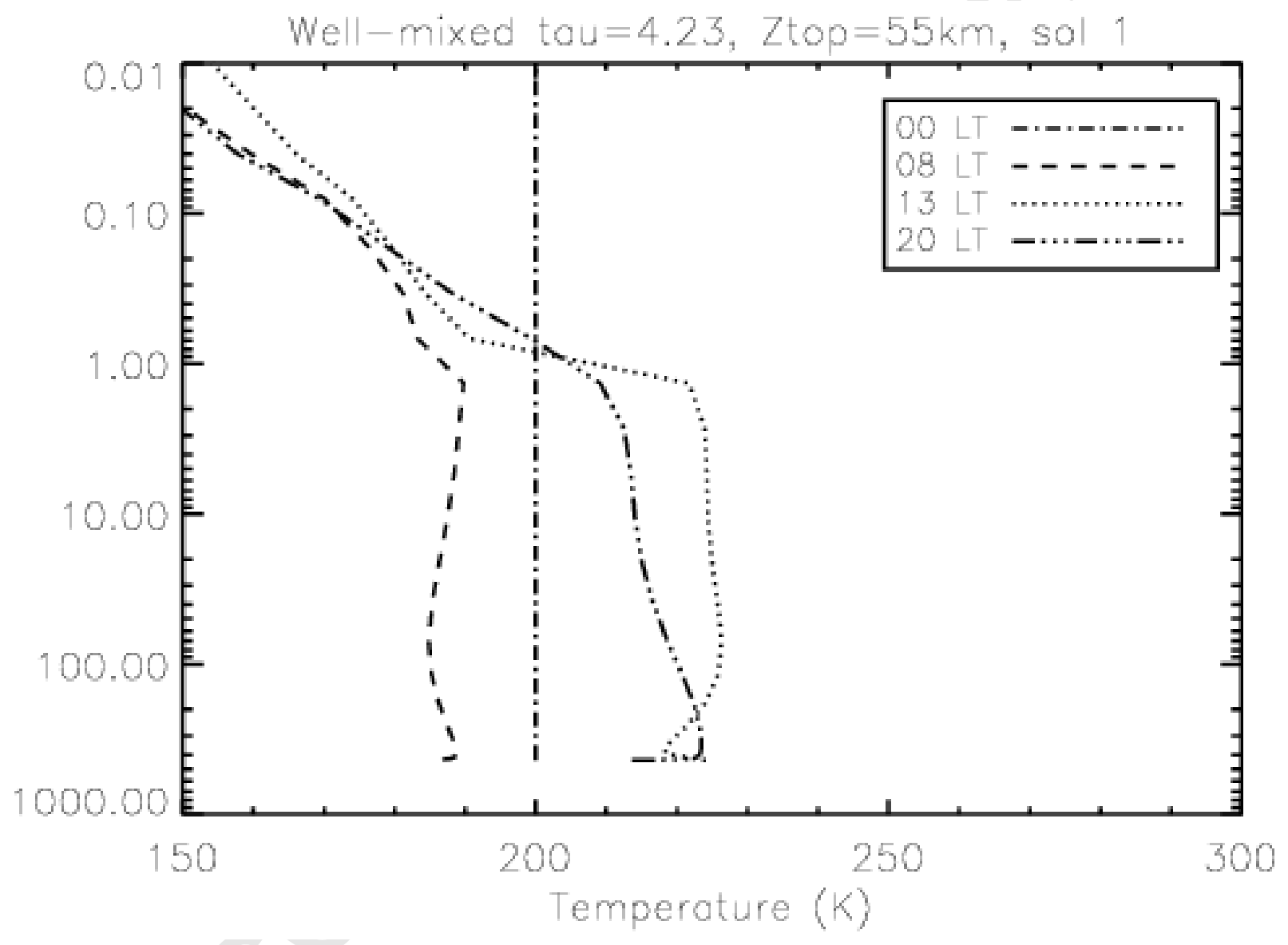

